\title{
The impact of graphene oxide sheet lateral dimensions on their pharmacokinetic and tissue distribution profiles in mice
}

Dhifaf A. Jasima, Leon Newman ${ }^{a}$, Artur Filipe Rodrigues ${ }^{a}$, Isabella A. Vacchib, Matteo A. Lucherelli $^{b}$, Neus Lozano ${ }^{a, c}$, Cécilia Ménard-Moyon ${ }^{b}$, Alberto Bianco ${ }^{b}$, Kostas Kostarelos ${ }^{a, c, *}$

a Nanomedicine Lab, National Graphene Institute and Faculty of Biology, Medicine \& Health, University of Manchester, AV Hill Building, Manchester M13 9PT, United Kingdom

b University of Strasbourg, CNRS, Immunology, Immunopathology and Therapeutic Chemistry, UPR 3572, 67000 Strasbourg, France.

c Catalan Institute of Nanoscience and Nanotechnology (ICN2), CSIC and BIST, Campus UAB, Bellaterra, 08193 Barcelona, Spain

* Correspondence to:

kostas.kostarelos@manchester.ac.uk 


\begin{abstract}
1 Abstract
Although the use of graphene and 2-dimensional (2D) materials in biomedicine has been explored for over a decade now, there are still significant knowledge gaps regarding the fate of these materials upon interaction with living systems. Here, the pharmacokinetic profile of graphene oxide $(G O)$ sheets of three different lateral dimensions was studied. The GO materials were functionalized with a PEGylated DOTA $(1,4,7,10$ tetraazacyclododecane-1,4,7,10-tetraacetic acid), a radiometal chelating agent for radioisotope attachment for single photon emission computed tomography (SPECT/CT) imaging. Our results revealed that $\mathrm{GO}$ materials with three distinct size distributions, large (l-GO-DOTA), small (s-GO-DOTA) and ultra-small (us-GO-DOTA), were sequestered by the spleen and liver. Significant accumulation of the large material (I-GO-DOTA) in the lungs was also observed, unlike the other two materials. Interestingly, there was extensive urinary excretion of all three GO nanomaterials indicating that urinary excretion of these structures was not affected by lateral dimensions. Comparing with previous studies, we believe that the thickness of layered nanomaterials is the predominant factor that governs their excretion rather than lateral size. However, the rate of urinary excretion was affected by lateral size, with large GO excreting at slower rates. This study provides better understanding of 2D materials behaviour with different structural features in vivo.
\end{abstract}

Key Words: Graphene oxide; functionalization; pharmacokinetics; nanomedicine, pharmacology 


\section{Introduction}

Graphene and related flat-shaped materials exhibit outstanding properties generated from their unique 2D geometry (1-3). These materials have attracted great interest from different scientific disciplines (1-3). The biomedical applicability of graphene has only been researched for the last decade (4). The available graphene surface area is the largest for any material at the nanoscale, which provides a potential delivery platform for maximum payload of therapeutic molecules and for bio-functionalization with imaging probes (5-8). The electrical, electronic (9), mechanical, optical properties $(8,10,11)$ and flexibility of graphene based materials $(2,12)$ allow their use as biosensing platforms and offer a great potential for use in regenerative medicine (13) and electroresponsive drug therapy . It also can be useful to meet the requirements of the electroactive nervous and cardiac systems and therefore provide a means of neuronal and cardiac drug delivery (14). All these properties offer interesting possibilities of graphene materials following their interaction with soft biological matter $(11,15)$.

Due to the great potential offered by using graphene materials for biomedical applications, it is critical to understand their fate in vivo (16). Graphene oxide (GO) has expanded the applications of graphene-based materials in biomedicine due to its hydrophilicity and improved compatibility with biological systems. GO has been administered intravenously (17-19), intraperitoneally, orally (20) and intravitreally (21) with no reported toxic effects even after long exposure times $(20,21)$. After intravenous or intraperitoneal administration, GO has been reported to accumulate in the mononuclear phagocytic system, or as more commonly known the reticuloendothelial system (RES). The spleen has been reported as the main site for the in vivo degradation of intravenously injected functionalized graphene (22). GO materials have also been reported to accumulate in the lungs $(23,24)$. Extensive urinary excretion of GO has been reported in several studies after intravenous (i.v.) injection of functionalized GO sheets in mice (16, 20, 25-29).

Existing studies have used very different types of $\mathrm{GO}(30,31)$ that can result in significantly different biological interactions. These interactions will depend on the type of surface functionalization, functional surface groups and dimensions of the GO sheets (17). The most popular administration route used in the preclinical development of materials for biomedical applications has been the intravenous (i.v.) route, which provides $100 \%$ bioavailability and therefore maximum information for tissue exposure and toxicity (32). Many studies have used imaging for studying the biodistribution of graphene materials by adding a labelling tag using single photon computed tomography (SPECT/CT) with gamma $(\gamma)$ emitting isotopes $(28,33)$, positron emission computed tomography 
1 (PET/CT) with positron emitting isotopes $(27,34)$ or even fluorescence tags for optical imaging $(26,34)$.

Previously we have demonstrated that the thickness of the graphene material plays a major role in its tissue accumulation and excretion (27), however, no systematic correlation with the GO sheet lateral dimension has been offered. In this study, three highly purified and well characterised graphene oxide materials that differ only in their lateral dimensions were functionalized with a chelating moiety for imaging purposes, namely a DOTA molecule tethered to a polyethylene glycol (PEG) linker. The resulting functionalized materials had three distinct sizes as well (I-GO-DOTA, s-GO-DOTA and usGO-DOTA). Then, whole-body imaging by SPECT/CT and full pharmacokinetic studies were carried out following i.v. administration of the GO-DOTA materials, coupled with analytical and histopathological analysis of critical organs. This study provided important information on the future design of graphene materials for possible tissue targeting applications.

\section{Results}

Preparation and characterisation of GO and GO-DOTA materials. GO was prepared by a modified Hummers' method under pyrogen-free conditions, as previously described (35-37). Morphology of GO sheets was characterised by DLS, TEM, and AFM (Figure 1). DLS revealed clear differences in the size distribution curves of the aqueous suspensions of the three types of GO materials (I-GO, s-GO and us-GO). The sonication employed to generate the different lateral size nanosheets (s-GO and us-GO) did not significantly impact their surface charge (Figure 1A-B).

In order to perform biodistribution studies, the GO materials were functionalized with the chelating agent DOTA, which was attached to a tetra-ethylene glycol [(PEG) $\left.{ }_{4}\right]$ molecule bearing a free amine group [DOTA(PEG) ${ }_{4}-\mathrm{NH}_{2}$ ], as described in Scheme 1. The functionalized chelating moiety [DOTA $(P E G)_{4}-\mathrm{NH}_{2}$ ], is referred to as 'DOTA' for ease throughout the manuscript. Although this process resulted in a decreased nanosheet surface charge (Figure 1B), DOTA functionalization did not significantly impact the colloidal stability of GO, in agreement with previous observations in physiological media (28). DLS is not particularly suitable as a technique to determine the dimensions of nonspherical particles (38) and we were not able to obtain reliable DLS data for the DOTAfunctionalized material. We emphasised on the structural characterisation of GO sheets before and after DOTA functionalization, performed by TEM and AFM (Figure 1C-D). The tested materials were composed of sheets with distinct differences in lateral dimensions (i.e. the longest dimension observed of each 2D sheet), which are described in detail in Table S1.

In summary, l-GO is comprised of sheets with lateral dimension between 1 and 35 $\mu \mathrm{m}$, whilst s-GO sheets range between $30 \mathrm{~nm}$ and $1.9 \mu \mathrm{m}$. The smallest material (us-GO) was characterised by a narrower size distribution, with lateral dimensions ranging from 10 $\mathrm{nm}$ to $550 \mathrm{~nm}$. Upon functionalisation there was a reduction in the sheet lateral dimension, particularly l-GO-DOTA compared to I-GO, where there was a 5-fold reduction (Table S1). 
Nevertheless, the lateral dimensions of s-GO-DOTA and us-GO-DOTA sheets remained markedly different from l-GO-DOTA and from each other. These differences could be attributed to the ring opening reaction attacking cooperatively aligned epoxides present in the GO sheet surface, which ultimately create fracture points $(39,40)$. AFM demonstrated that the thickness of all GO materials had increased following DOTA functionalisation (Table S1). This could be due to the addition of functional groups and has been observed previously in several studies $(20,28,41)$.

The samples were also characterised by X-ray photoelectron spectroscopy (XPS) (Table S1 and Figure S1-3). The XPS survey table shows 30-31\% oxygen content in all three starting materials. The oxygen content is slightly lower after functionalisation with DOTA, with the introduction of $1.1-1.4 \%$ nitrogen content, due to the addition of the DOTA molecule. Similarly, in the oxygen O1s high resolution spectra, there is a clear increase in percentage of the carbonyl peak (Figure S2). This is due to the introduction of carboxylic acids and amides. On the other hand, the carbon $\mathrm{C} 1 \mathrm{~s}$ high resolution spectra (Figure S1) are more complex and revealed changes between the ratio of the carbon region and the carbon-oxygen region. This is often the case after any kind of treatment of GO $(42,43)$ due to the loss of some labile oxygenated functional groups. In particular, the component attributed to $\mathrm{C}-\mathrm{O}-\mathrm{C}$ bonds decreased whereas the $\mathrm{C}-\mathrm{OH} / \mathrm{C}-\mathrm{N}$ band increased. These changes suggest that the reaction of epoxide ring opening occurred, thus generating $\mathrm{C}-\mathrm{OH}$ groups, alongside the introduction of amino groups from the DOTA compound, as previously reported (44). However, the C-O-C peak did not disappear because of the ether groups in the PEG chain. Two components could be identified in the nitrogen N1s high resolution spectra, namely the amine/amide and the ammonium peaks at $\sim 400 \mathrm{eV}$ and $402 \mathrm{eV}$, respectively. Both amines and amides are present in the DOTA molecule, further indicating the successful functionalisation of GO with the DOTA moiety (Figure S3).

Thermogravimetric analysis (TGA) also validated chemical functionalisation of GO (Figure S4). GO starts to lose mass at temperatures even lower than $100^{\circ} \mathrm{C}$ due to residual water molecules adsorbed on the GO sheets $(37,43,45,46)$. The two weight losses above $150^{\circ} \mathrm{C}$ are due to the oxygenated species present on the surface of $\mathrm{GO}$ and to the DOTA molecule in the case of the functionalized materials. The main weight loss occurs at lower temperature (around $220^{\circ} \mathrm{C}$ ) for the three GO-DOTA compared to the starting materials $\left(\sim 240^{\circ} \mathrm{C}\right)$ was considered another indication of covalent functionalization of the DOTA functionality. The \%N obtained by XPS indicated that the three conjugates have a similar DOTA loading. Taken together, these results show that the functionalization of GO with the DOTA derivative was successful and did not cause significant reduction of the starting material.

\section{Efficiency and purity of radiolabeling of $\left[{ }^{111}\right.$ In]GO-DOTA. The efficiency of} radiolabelling of the three types of GO $\left(\left[{ }^{111} \mathrm{In}\right] \mathrm{l}-\mathrm{GO}-\mathrm{DOTA},\left[{ }^{111} \mathrm{In}\right] \mathrm{s}-\mathrm{GO}-D O T A\right.$ and $\left[{ }^{111} \mathrm{In}\right] \mathrm{us}$ GO-DOTA) was compared to control [ $\left.{ }^{111} \mathrm{In}\right]$ DOTA in Figure 2A. The radiolabelling efficiency of the three samples was $\sim 70 \%$ at the application point. The samples were purified by removal of unbound [ ${ }^{111} \mathrm{In}$ ]DOTA by centrifugation, reaching a purity of $\sim 90 \%$ after centrifugation (Figure 2B).

Stability of radiolabeling of [111In]GO-DOTA. The three samples had insignificant variable stability in PBS at $37^{\circ} \mathrm{C}$ up to 1 week as shown in Figure $\mathbf{2 C}$, while the samples were stable and retained their radiolabelling purity in $50 \%$ serum as shown in the same figure and in Figure S5. The latter figure demonstrated that the three samples retained a 
signal at the application point of the TLC compared to the control materials alone $\left[{ }^{111} \mathrm{In}\right]$ EDTA and [ ${ }^{111} \mathrm{In}$ ]DOTA which moved to the solvent front.

Pharmacokinetics and tissue distribution after i.v. administration. The biodistribution and excretion of the three types of GO ([ $\left[{ }^{11} \mathrm{I} n\right] \mathrm{l}$-GO-DOTA, $\left[{ }^{111} \mathrm{In}\right] \mathrm{s}-\mathrm{GO}-D O T A$ and $\left[{ }^{111} \mathrm{In}\right] \mathrm{us}-$ GO-DOTA) after i.v. administration was studied by SPECT/CT imaging and cut-and-count Y-scintigraphy (Figure 3, Figure 4 and Figure S6-9). The levels in blood determined by $\mathrm{Y}^{-}$ counting are shown in Figure 3A. The curve demonstrated that pharmacokinetics for all materials followed two-compartment first order kinetics. The pharmacokinetic parameters are summarised in Table $\mathbf{S 2}$ and the values remaining in blood after $1 \mathrm{~h}$ are compared to $24 \mathrm{~h}$ in Table S3. All three materials were removed from blood very rapidly with only less than $1 \%$ of the injected dose remaining in the blood after $24 \mathrm{~h}$. Dynamic SPECT/CT imaging was carried out during the i.v. administration of the materials (Figure S6). The first panel shows the 2 min phase of injection (movie can be played online), showing the delivery from the tail vein and through the vena cava then through whole blood. All three materials ([$\left[{ }^{11} \mathrm{I} \mathrm{n}\right] \mathrm{l}-$ GO-DOTA, $\left[{ }^{111} \mathrm{In}\right] \mathrm{s}$-GO-DOTA and [ $\left.\left.{ }^{111} \mathrm{In}\right] \mathrm{us}-\mathrm{GO}-\mathrm{DOTA}\right)$ and the control $\left[{ }^{111}\right.$ In]DOTA start to accumulate in the organs within the first hour as demonstrated in the planer images in the second panel in the same figure. Organ accumulation is very evident after $4 \mathrm{~h}$ and $24 \mathrm{~h}$ (last two panels, Figure S6). Time activity curves for each material are presented in Figure S7, indicating a huge accumulation of the larger material $\left(\left[{ }^{111} \mathrm{In}\right] \mathrm{l}-\mathrm{GO}\right.$ DOTA) in the lungs, while the other two materials accumulated mainly in the liver and spleen. All three materials presented bladder and kidney signals at early time points. The control sample [ $\left.{ }^{111} \mathrm{In}\right]$ DOTA was totally excreted after $1 \mathrm{~h}$ with huge bladder signal compared to the other materials (Figure S7).

These results were further confirmed by a separate experiment using 3D SPECT/CT for better image resolution as demonstrated in Figure 3B and Figure S8 for the first batch of mice and Figure $\mathbf{S 9}$ for a second batch of mice. The scale bars are expressed in percentage of injected dose (\%ID) per gram of tissue in Figure 3B and Figure S9, while it is expressed in $\mathrm{MBq}$ in Figure $\mathbf{S 8}$ and Figure $\mathbf{S 1 0}$. These images show minor intestinal signals in all mice. Expression in \%ID per gram of tissue will show the minute amounts in blood levels in light organs. The total remaining amounts in the whole body after $24 \mathrm{~h}$ quantified from the SPECT images are presented in Table S4, with $50.8 \%$, $36.5 \%$ and $38.1 \%$ for $\left[{ }^{111} \mathrm{In}\right] \mathrm{l}-\mathrm{GO}-\mathrm{DOTA},\left[{ }^{111} \mathrm{In}\right] \mathrm{s}-\mathrm{GO}-D O T A$ and $\left[{ }^{111} \mathrm{In}\right]$ us-GO-DOTA, respectively.

The whole-body SPECT/CT imaging data were validated by a separate cut and count experiment counting the \%ID per whole organ of [ $\left.{ }^{111} \mathrm{In}\right] \mathrm{GO}-\mathrm{DOTA}$ or \%ID per gram of tissue measured by $\mathrm{Y}$-scintigraphy in a separate experiment with at least 4 mice per condition (Figure 3C). The data confirmed the same pattern of the SPECT/CT data with lung accumulation for the largest material ([111 In]l-GO-DOTA) and liver and spleen accumulation for the all three materials ([111 In]l-GO-DOTA, $\left[{ }^{111} \mathrm{In}\right] \mathrm{s}-$-GO-DOTA and [111In]us-GO-DOTA). Some kidney and bladder signals were detected at early time points, while minute intestinal signal was evident at the later time points. Very little signals were detected in the control sample (Figure $\mathbf{3 C}$, bottom row).

Urinary and fecal excretion. Urinary and faecal excretion was studied by collecting the urine and faeces of injected mice to further examine the extent of elimination of the three materials (Figure 4). Pooled urine samples $(n=4)$ were collected and counted for 
radioactivity at different time points (Figure 4A). The [ $\left.{ }^{111} \mathrm{In}\right]$ us-GO-DOTA showed the maximum excretion compared to the other two samples. Furthermore the samples were analysed by Raman spectroscopy as shown in Figure 4B. The Raman signature of GODOTA was detected in the urine of all three materials, confirming the excretion of the material regardless of the lateral size. Pooled faecal samples were collected after $24 \mathrm{~h}$ and measured for radioactivity, all samples indicated faecal excretion (Figure 4C).

Histopathology after i.v. administration of the three materials. Tissue samples (lung, liver, spleen and kidneys) were examined for histopathology using $H$ \& $E$ (haematoxylin and eosin) staining of paraffin embedded tissue sections for two animals per condition (Figure 5 and Figure S10-15). Lungs of mice injected with all the GO materials demonstrated an interesting distribution pattern (Figure 5 and Figure S10). All GO materials were predominantly detected as agglomerates within the lumen of lung blood vessels, which indicates the retention of GO in the lung capillaries as a size-dependent phenomenon, with the I-GO-DOTA material showing the largest, and the most agglomerates, as quantified in Figure $\mathbf{S 1 1}$ and shown in different regions in Figure $\mathbf{5}$ and Figure S10. In the case of the mice spleen samples, no evidence of histopathology was determined in the red pulp (second panel) and the white pulp (third panel) in any of the samples as compared to the controls (Figures S12-13). Liver tissue (Figure S14) and kidney tissue (both glomerular and tubular regions) (Figure S15) also demonstrated healthy anatomical structures with no evident histopathology in any of the samples.

\section{Discussion}

To date there is no direct method to quantitatively measure the amount of GO in physiological fluids (e.g. tissues, blood and urine) due to the background interferences from the complex molecular composition of such biological fluids. Studies rely on labelling of the material for quantification, though other methods can offer qualitative detection such as TEM and Raman spectroscopy. In this work, we studied the pharmacokinetic profile of three lateral sizes of thin GO sheets. The functionalisation of GO with PEGylated DOTA was performed to allow the chelation of a radioactive metal $\left[{ }^{111} \mathrm{In}\right]$ for studying and quantifying the tissue distribution of the materials. The functionalisation of $\mathrm{GO}$ with the PEGylated DOTA was performed via epoxide opening. Because of the abundance of epoxides on the GO surface, this strategy generally leads to higher levels of functionalization compared to the derivatisation of hydroxyls or carboxylic acids (47).

Structural characterisation was carried out by TEM and AFM to reveal whether GO sheets would have marked differences in their morphology. The materials were produced with controlled lateral dimensions by tuning their lateral size by sonication as previously reported $(37,48-50)$. After functionalization all three materials maintained their $2 \mathrm{D}$ morphology, while the thickness increased from single to a few layers following DOTA functionalization due to the presence of the functional groups and minor agglomeration that led to slightly thicker sheets. These findings are consistent with previous reports of functionalization of GO with DOTA $(27,28)$ PEG $(20,51)$, dextran $(25)$ and bovine serum albumin (52). Interestingly, we noticed that l-GO underwent a marked reduction in sheet lateral dimension after DOTA functionalization. Surface functionalization of GO has also been reported to reduce the lateral dimension of the sheets $(28,52)$. However, the lateral 
dimensions of all functionalized GO-DOTA materials remained markedly different from each other.

The analysis of the XPS spectra (Table S1 and Figures S1-3) showed that the functionalization of GO with the DOTA derivative was successful, as demonstrated by the detection of nitrogen in the GO-DOTA materials compared to their starting counterparts. Moreover, nitrogen atoms were detected in the form of amines and amides (Figure S3), which are present in the DOTA molecule. The higher intensity of the carbonyl peak in the carbon high resolution spectra after functionalization (Figure S1) is due to the carboxylic acids of the DOTA molecule and it is also an indication of the presence of amides. On the other hand, the changes in the carbon high resolution spectra after functionalization are more subtle. First, there is a decreased abundance of oxidised carbon atoms compared to the graphitic carbon region, due to the loss of some labile groups after functionalization of $\mathrm{GO}$. The contribution of the epoxides $(\mathrm{C}-\mathrm{O}-\mathrm{C})$ is reduced compared to the $\mathrm{C}-\mathrm{OH} / \mathrm{C}-\mathrm{N}$ band, which is coherent with the introduction of the DOTA moiety by opening of epoxides, thus generating $\mathrm{C}-\mathrm{OH}$ and introducing amino groups. Chemical functionalization of $\mathrm{GO}$ was further evidenced by analysing the different GO derivatives by TGA under an inert atmosphere (Figure S4). GO is thermally unstable and starts to lose mass at temperatures even lower than $100^{\circ} \mathrm{C}$ due to residual water molecules adsorbed on the GO sheets. A main weight loss was identified at $230^{\circ} \mathrm{C}$ that is due to the elimination of labile oxygencontaining groups (53). All GO-DOTA materials revealed a lower thermal stability probably due to functionalization with DOTA. Therefore, taken together these results confirm the effective grafting of DOTA on the surface of the three GO samples. In addition, the structure of $\mathrm{GO}$ is preserved and the functionalization strategy induced no significant reduction, thus enabling to obtain stable suspensions in aqueous solutions.

Other studies have reported radiolabelling of GO materials with iodine resulting in iodinated constructs that are unstable, while the high affinity of iodine for the thyroid gland can be misleading with regards to 2D material biodistribution profiles (25). Radiolabelling with ${ }^{111}$ In has been performed previously using physical adsorption of the DTPA (diethylenetriaminepentaacetic acid) chelating agent on the surface of $\mathrm{GO}$ by $\pi$-stacking. Such strategy however increased the thickness of the GO sheets dramatically (33). The radiolabelling efficiency and stability of covalently bound DOTA to GO materials has been tested before with high efficiency and stability $(27,28)$. In this study a similar strategy was exploited using GO materials with three different lateral dimensions (I-GO-DOTA, s-GODOTA and us-GO-DOTA). The radiolabelling efficiency was not affected dramatically by the lateral size of the GO sheets. All samples remained stable up to $24 \mathrm{~h}$ at $37^{\circ} \mathrm{C}$ in $50 \%$ serum. Similar to original samples without serum incubation, the control [ $\left.{ }^{111} \mathrm{In}\right]$ DOTA travelled to the solvent front as compared to the three samples that remained at the bottom of the TLC (Figure S5). This indicated that the [ ${ }^{111}$ In]DOTA was attached to the GO materials with no interferences occurring from the serum proteins at the times that were tested (reflecting the time predicted for the material will spend in blood after injection and beyond that time). This avoids any possible conflictions in the tissue distribution that are created from the detachment of the [ ${ }^{111}$ In]DOTA. Therefore the purity of material was confirmed to be suitable for in vivo administration. Furthermore the in vivo stability was confirmed by the Raman signal that coregistered with the radioactivity at the bottom of the TLC strips in the urine of the mice after injection as compared to the control free label that was predominately at the top of the TLC strips (Figure 4B). This was comparable to the original samples before injection in Figure 2B this further confirmed the stability and association of the label with the GO material even after injection. 
Compared to our previous studies (27-29) where the small and thin GO-DOTA material was only injected, in this study, three materials with comparable thickness and the three different sizes were injected intravenously in mice. All three materials were removed from blood within minutes, as shown by the first-phase distribution half-life $\left(t_{1 / 2 \alpha}\right)$. Although no significant differences were seen in the pharmacokinetic data of the materials, the $\left[{ }^{111} \mathrm{In}\right.$ ]DOTA control was removed from blood faster. In the second phase the $\left[{ }^{111} \mathrm{In}\right]$ us-GODOTA material was more similar to $\left[{ }^{111} \mathrm{In}\right]$ DOTA control and remained slightly longer in circulation in the second phase half-life $\left(t_{1 / 2 \beta}\right)$. The amounts remaining in blood after $1 \mathrm{~h}$ and $24 \mathrm{~h}$ were more elevated for the $\left[{ }^{111} \mathrm{In}\right] \mathrm{s}-\mathrm{GO}-D O T A$ and $\left[{ }^{111} \mathrm{In}\right]$ us-GO-DOTA compared to [ $\left.{ }^{111} \mathrm{In}\right] \mathrm{l}-\mathrm{GO}-\mathrm{DOTA}$ (Figure 3A, Figure S5,Table S2 and Table S3). Smaller nanoparticles are well known to circulate longer $(54,55)$. The area under the blood concentration time curve (AUC), steady state volume of distributions $\left(\mathrm{Vd}_{\mathrm{ss}}\right)$ (indicating the body overall and tissue exposure) and the clearance values are shown in Table S2. The $\left[{ }^{111}\right.$ In]DOTA control was almost entirely removed from blood at the early hours after administration (Table S3). On the contrary, the remaining amounts in the whole body after $24 \mathrm{~h}$ for the three materials was much higher compared to control material (Table S4).

It is clear from our results that tissue distribution occurs very rapidly for all three materials and is largely affected by the size of the graphene sheet (Figure 3B-C and Figure S6-9). It is very evident that the large material ([111 In]l-GO-DOTA) tends to accumulate in the lungs early after injection, with reductions in the signal after $24 \mathrm{~h}$ as seen by both the time activity curve and the $\mathrm{Y}$-counting experiment (Figure S7 and Figure $3 \mathbf{C}$, respectively). Larger nanomaterials tend to accumulate in the lungs after i.v. administration due to the first capillary bed (55). This has also been demonstrated with graphene materials $(17,56,57)$. Some material also remained in the lung tissues after $24 \mathrm{~h}$ as seen in the SPECT/CT data, $\mathrm{Y}$-counting and in the $\mathrm{H} \& \mathrm{E}$ sections/semi-quantification (Figure 3B-C, Figure S6-9 and Figure S11). Some thickening of the alveolar walls was also seen especially in lungs from mice injected with I-GO-DOTA and s-GO-DOTA, indicating cellular infiltration and constriction, probably as a result of the material entrapment in the lung tissues. We observed a clear reduction of the lung signal after $24 \mathrm{~h}$. It is known that the removal of nanomaterials from the lung is carried out by phagocytic uptake of the lung macrophages. This is the main mechanism to remove the insoluble aggregated nanoparticles into the micrometer-sized particles from the lung tissues. Particle-containing macrophages may re-enter into the interstitium and be cleared by the lymphatics or other organs (58).

The smaller materials ([111 In]s-GO-DOTA and [ $\left.{ }^{111} \mathrm{In}\right]$ us-GO-DOTA) accumulated mainly in the liver and spleen with slight variability between animals in maximum accumulations in these organs. The $\left[{ }^{111} \mathrm{In}\right]$ us-GO-DOTA showed a slight reduction in the hepatic signal after $24 \mathrm{~h}$ compared to the $\left[{ }^{111} \mathrm{In}\right] \mathrm{s}$-GO-DOTA that remained the same. On the other hand, both materials accumulated in the spleen at high concentration even after 24h (Figure 3B-C and Figures S6-9). It is well known that nanomaterials, including graphene-based materials, get trapped in the mononuclear phagocytic system (RES) (16, $25,34,59,60)$. This happens within the liver due to the non-continuous liver endothelia with vascular fenestrations measuring $50-100 \mathrm{~nm}$, leading to nonspecific accumulation of nanoparticles within this range. In the spleen, interendothelial cell slits with a size range of 200-500 nm mediate the retention of particles $>200 \mathrm{~nm}$ (55). Particle shape also accounts for accumulation in certain tissues, with elongated nanoparticles mainly in the spleen, while the more spherical ones accumulate in the liver (61). The total amount of material 
remaining in the body of mice injected with the $\left[{ }^{111} \mathrm{In}\right] \mathrm{l}-\mathrm{GO}-\mathrm{DOTA}$ was the highest compared to the other two materials (Table S3), which can be explained by the fact that larger and thicker materials are more difficult to be cleared from the body (27).

Though there was slight variability between animals, the bladder measurements obtained from the $\mathrm{y}$-counting experiments are largely dependent on the urine content in the bladder that can largely vary. But, it is clear from the data obtained herein that the graphene sheets were excreted through urine regardless of their lateral dimension (Figure 3B-C, Figure 4A-B, Figures S6-9). The Raman signature of GO-DOTA was detected in the urine of injected mice confirmed the presence of intact sheets undergoing urinary excretion. The kidney and bladder profiles in Figure 3B-C and Figure S6 suggested that the larger sheets ([111In]l-GO-DOTA) were excreted at a slower rates compared to the other two materials ([$\left[{ }^{111} \mathrm{In}\right] \mathrm{s}-\mathrm{GO}-D O T A$ and $\left[{ }^{111} \mathrm{In}\right]$ us-GO-DOTA). Though, there could be a possibility of a very small fraction of small sheets in all the three GO-DOTA samples because of population of size distributions, however previous studies by our group and others gave direct evidence of the excretion of much larger sheets than the kidney glomerular filtration cut off due to their thin and flexible nature $(29,62)$. All materials had a delayed excretion profile compared to the control probe, which was eliminated almost entirely instantly. Gamma counting of pooled urine samples can sometimes be misleading due to huge loss on the surfaces of the metabolic cages. Therefore, comparing the quantities in urine to the control $\left(\left[{ }^{11}\right.\right.$ In $]$ DOTA $)$ chelator sample is more realistic as the chelating agents are predicted to be excreted almost entirely with minimal tissue accumulation (63). We observed that the quantities excreted were much higher for the usGO-DOTA compared to the other two samples ([ $\left.{ }^{111} \mathrm{In}\right] \mathrm{l}-\mathrm{GO}-\mathrm{DOTA}$ and $\left.\left[{ }^{111} \mathrm{In}\right] \mathrm{s}-\mathrm{GO}-\mathrm{DOTA}\right)$ and close to the excretion of the control [ $\left.{ }^{111} \mathrm{In}\right] \mathrm{DOTA}$. Overall, these results show that all three materials were excreted and the smaller material was excreted faster and to a larger extent.

We and others have demonstrated previously that large, thin functionalized GO sheets can cross the glomerular filtration barrier (GFB) although they have dimensions that exceed the GFB cut off $(16,27-29,62)$. However, this is the first time we demonstrate urinary excretion of large GO sheets. Our histological data (Figure S16) resonates with our previous results, illustrating the lack of damage to kidney regions. This suggested that excretion of GO sheets occurred as a passive mechanism regardless of their size (provided they are thin and flexible enough), possibly due to their morphological reconfiguration of the sheets either by sliding, squeezing, rolling or folding (29).

The small intestinal signals in the SPECT/CT images at $24 \mathrm{~h}$ and values in faeces suggested slight faecal excretion, which could occur by biliary excretion, similarly to previous findings with PEGylated GO (16), which bared PEG moieties functionalized GODOTA materials. No damage to organs (spleen, liver and kidneys) was determined by our histological $\mathrm{H} \& \mathrm{E}$ examinations for all three materials, consistent with many other previous studies $(16,20,64,65)$. However, lung sections indicated some thickening of the alveolar walls especially for the large material (Figure 5, S11 -12). However, all three GO-DOTA materials were detected as agglomerates within the lumen of the lung blood vessels (Figure S12) and the retention of material in the lung capillaries was a size-dependent phenomenon. This retention and lung histopathological changes that can further lead to fibrosis and lung damage at later time points have been observed in other studies using large and non-functionalized materials $(56,66,67)$ and requires further investigations. 
1 This work suggests that the lateral dimension of the graphene material is critical to 2 determine the fate after i.v. administration. The multi-phase biodistribution profile of the 3 GO sheets studied here can be due to the wide size distribution of the GO sheets. We 4 envisage that the thin, flexible and small sheets tend to cross the glomerular filtration 5 barrier, while the larger and thicker sheets are preferentially entrapped in the lung. The 6 remaining smaller sheets were taken up by the liver and spleen cells. DOTA chelator with high chemical and radiolabelling stability regardless of the GO starting lateral dimension. Intravenous administration of the GO-DOTA constructs led to rapid and significant uptake by the lungs of the larger material, while the smaller materials targeted the liver and spleen. All three radiolabelled GO-DOTA materials were excreted regardless of their lateral dimension, indicating the possibility that the thickness rather than size may be the major factor that governs the ability of GO to be excreted. Nevertheless, the lateral dimension affected the rate of excretion. These findings provide further insight on the kinetics and biodistribution of thin functionalized GO sheets with different lateral dimensions after intravenous administration in mice. This has important implications in the future design of graphene-based materials for biological applications and for targeting of different organs. Indeed, large GO could be exploited for lung delivery, whereas smaller dimensions could target the liver and spleen. Ultra-small GO is excreted to a larger extent, which would make it attractive for imaging applications such as dynamic imaging where the contrast agent needs to exit the body quickly after the imaging session, this facilitates pharmacokinetic testing and reduces the number of animals required if used as a carrier for image contrast agents (68). Due to the high surface area it could further be used to deliver imaging probes and therapeutics simultaneously for thaeranostic applications. Future studies are necessary to further assess the safety profile of graphene-based nanomaterials and warrant direct quantitative detection techniques of the materials in the complicated biological media for further validation. 


\section{Experimental}

Chemical synthesis of GO. All reagents used in the production of GO were purchased from Sigma Aldrich (UK), except water for injections, which was obtained from Dutscher Scientific (UK). GO was produced by a modified Hummers' method under endotoxin-free conditions (35-37). All glassware were depyrogenated prior adding the mixture of $0.8 \mathrm{~g}$ of graphite flakes (Graflake 9580 , kindly provided by Nacional de Grafite Ltda, Brazil) with $0.4 \mathrm{~g}$ of sodium nitrate in a round-bottom flask that was placed in an ice bath, followed by the slow addition of $18.4 \mathrm{~mL}$ of $99.999 \%$ sulphuric acid. When the mixture became homogenised, $2.4 \mathrm{~g}$ of potassium permanganate was slowly added for $30 \mathrm{~min}$. The dropwise addition of $37.5 \mathrm{~mL}$ of water for injections resulted in a violent exothermic reaction, after which the temperature of the mixture was carefully maintained at $98^{\circ} \mathrm{C}$ for $30 \mathrm{~min}$. After the addition of $112.5 \mathrm{~mL}$ of water for injection, the reaction was stopped with $12 \mathrm{~mL}$ of $30 \%$ hydrogen peroxide, which reduced the unreacted potassium permanganate and manganese-based intermediate products to manganese sulphate salts. The resulting graphite oxide suspension underwent a series of exfoliation and centrifugation steps at $9000 \mathrm{rpm}$ for $20 \mathrm{~min}$, where the supernatant was replaced by fresh water for injections, until the $\mathrm{pH}$ of the supernatant became neutral and a brownish gel-like layer was formed on the top of the pellet. Warm water for injections was used to extract l-GO flakes from the graphite pellet. In order to produce s-GO flakes, I-GO underwent a 5-min sonication step in a bath sonicator (VWR, UK) operating at $80 \mathrm{~W}$, which exfoliated and broke down the micrometre-sized flakes. The exfoliated suspensions were centrifuged at $13000 \mathrm{rpm}$ for $20 \mathrm{~min}$ at $20^{\circ} \mathrm{C}$, and the respective supernatants were carefully extracted, containing only the small nanometre-sized flakes. For production of the us-GO flakes, the I-GO underwent a similar process, involving a 4-hour sonication step followed by centrifugation at $13000 \mathrm{rpm}$ for $1 \mathrm{~h}$ at $20^{\circ} \mathrm{C}$.

Preparation of GO-DOTA materials. $\mathrm{NH}_{2}-\mathrm{PEG}_{4}$-DOTA (CheMatech) $(9 \mathrm{mg}, 0.0129 \mathrm{mmol}$ ) was added to an aqueous suspension of GO starting materials (us-GO, s-GO or l-GO) (9 mg, $1 \mathrm{mg} / \mathrm{mL}$ ). The mixture was left to react for 2 days under continuous stirring at room temperature. GO-DOTA was then directly dialyzed in MilliQ water for 4 days. The final dialyzed GO-DOTA was stored at $7^{\circ} \mathrm{C}$ in water without further treatment.

Preparation of ${ }^{111}$ In labelled GO-DOTA and control DOTA materials. I-GO-DOTA, s-GO-DOTA, us-GODOTA and control material DOTA(PEG) $)_{4}-\mathrm{NH}_{2}$ referred in this manuscript as 'DOTA' for ease, were diluted with an equal volume of $0.2 \mathrm{M}$ ammonium acetate buffer $\mathrm{pH} 5.5$, to which $10-20 \mathrm{MBq}$ of ${ }^{111} \mathrm{InCl}_{3}$ (CURIUM ${ }^{\mathrm{TM}}$ previously, Mallinckrodt RP) was added, in separate experiments. The indium was left to react with the GODOTA and DOTA control for $60 \mathrm{~min}$ at $60^{\circ} \mathrm{C}$ with vortexing every $5 \mathrm{~min}$ after which the reaction was quenched by the addition of $0.1 \mathrm{M}$ EDTA chelating solution. The radiolabelling was carried out in several separate experiments at different occasions and with different operators.

Radiolabelling efficiency of GO-DOTA materials. To determine the labelling efficiency of each final product, aliquots were diluted five folds in PBS and then $1 \mu \mathrm{L}$ spotted on silica gel impregnated glass fibre sheets (PALL Life Sciences, UK). The strips were developed with a mobile phase of $50 \mathrm{mM}$ EDTA in $0.1 \mathrm{M}$ ammonium acetate and allowed to dry before analysis. The strips were developed with a mobile phase of 25 mM EDTA in $0.1 \mathrm{M}$ ammonium acetate and allowed to dry before analysis. This was then developed and the autoradioactivity counted using a Fujifilm fluorescence image analyser (FLA-3000 series, Tokyo, Japan). The immobile spot on the TLC strips indicated the percentage of radiolabeled GO-DOTA, while free $\left[{ }^{111}\right.$ In]DOTA(PEG)4-NH2, referred throughout this manuscript as [ ${ }^{111}$ In]DOTA for ease was seen as the mobile spots near the solvent front.

Radiolabelling stability of GO-DOTA materials. To determine the stability of the labeled [111In]GO-DOTA materials, aliquots of each final product were diluted five-fold either in PBS or $50 \%$ mouse serum and then incubated at $37^{\circ} \mathrm{C}$ over 1 week. At different time points (0h, 24h, 48h and 7 days), $1 \mu \mathrm{L}$ of the aliquots was spotted on silica gel impregnated glass fibre sheets and then developed, and quantified as described above.

TEM analysis. $20 \mu \mathrm{L}$ of sample $(200 \mu \mathrm{g} / \mathrm{mL})$ was dropped on a glow-discharged carbon-coated copper grid (CF400-Cu) (Electron Microscopy Services, UK). Filter paper (Merck-Millipore, UK) was used to absorb the unbound material. Samples were then observed with a FEI Tecnai 12 BioTWIN microscope (Techni, Netherlands) with an acceleration voltage of $100 \mathrm{kV}$. Images were taken using a Gatan Orius SC1000 CCD camera (GATAN, UK). Size using TEM distributions were performed as described previously $(37,49,50)$. 
AFM analysis. AFM was performed on freshly cleaved mica, treated with $40 \mu \mathrm{L}$ of poly-L-lysine (SigmaAldrich, UK) to present a positively charged surface, which facilitated the adhesion of the GO sheets. Aliquots of $10 \mu \mathrm{L}$ were then transferred of the respective suspensions onto the mica surface coated with poly-L-lysine and left to adsorb for $2 \mathrm{~min}$. Unbound GO sheets were then removed via gentle washing with $2 \mathrm{~mL}$ of MilliQ $\mathrm{H}_{2} \mathrm{O}$ and left to dry at room temperature. During analysis, a Multimode atomic force microscopy (Bruker, UK) was used in tapping mode in order to reduce damage to the samples for height (trace and retrace) and amplitude. Scans were completed using an Otespa tapping mode tip (Bruker, UK) using the following parameters: a scan rate of $1 \mathrm{~Hz}$; lines per scan of 512; an integral gain of 1 and a proportional gain of 5 ; an amplitude set point value of $150 \mathrm{mV}$ was maintained constant between all measurements. Scan areas were set at $2500 \mu \mathrm{m}^{2}, 400 \mu \mathrm{m}^{2}, 100 \mu \mathrm{m}^{2}$, and $25 \mu \mathrm{m}^{2}$. Post image processing was completed using the Bruker Nanoscope Analysis software (version 1.4, Bruker, UK). Size and thickness distributions were performed as described previously $(37,49,50)$.

XPS analysis. The three GO-DOTA aqueous suspensions were drop-casted on silicon wafers and dried overnight. XPS analysis was carried out with a Thermo Scientific K-ALPHA monochromatic photoelectron spectrometer with a basic chamber pressure of $10^{-8}-10^{-9}$ bar and an Al anode as X-ray source (1486 eV). A spot size of $400 \mu \mathrm{m}$ was selected. Every sample was analyzed three times. The survey spectra are an average of 10 scans taken with a pass energy of $200.00 \mathrm{eV}$ and a step size of $1 \mathrm{eV}$. The high resolution spectra are an average of 10 scans taken with a pass energy of $50.00 \mathrm{eV}$ and a step size of $0.1 \mathrm{eV}$. The pass energy of 50.00 $\mathrm{eV}$ corresponds to $\mathrm{Ag} 3 \mathrm{~d}_{5 / 2}$ line FWHM of $1.3 \mathrm{eV}$. A pass energy of $50.00 \mathrm{eV}$ for the high resolution spectra was applied because using lower pass energies has shown no improvement in FWHM for graphene materials on Thermo Scientific K-ALPHA. An electron flood gun was turned on during analysis as charge neutralizer. This electron flood gun was not totally efficient for charge compensation, thus an error due to charging was still identified in some samples.

Data analysis casaXPS (2.3.18) software was used. A Shirley background subtraction and charge correction were applied. No reference element was analysed, thus, the spectra were corrected to the C-C peak at 285.2$285.3 \mathrm{eV}$, if needed. Error due to this charge correction was taken into account. A line-shape $70 \%$ Gaussian/30\% Lorenzian [GL(30)] is selected for all peaks beside for $s p^{2} \mathrm{C}$ peak. For $s p^{2} \mathrm{C}$ peak an asymmetric line-shape was chosen $[A(0.4,0.38,20) G L(20)]$. FWHM was constrained to be the same for all peaks, beside the pi-pi* peak and the water peak because these are broad signals. The peak position for each bond was constrained to be the same in every spectra, with a low ratio of variability. Due to the difficulty to take standards for carbon nanomaterials, database and reference articles were used as peak reference value. For the carbon high resolution spectra almost every bond was considered individually, keeping into consideration the error due to the proximity of the binding energy $(\mathrm{BE})$ values and proportional to the overlapping ratio. Only $\mathrm{C}-\mathrm{OH} / \mathrm{C}-\mathrm{N}$ and $\mathrm{C}=\mathrm{OOH} / \mathrm{C}=\mathrm{ONR}_{2}$ were cumulated under the same peak due to the higher proximity of the $\mathrm{BE}$ values. For oxygen and nitrogen high resolution spectra all signals were assembled due to the high proximity of the BE values instead. Also with this approximation a certain degree of error has to be taken into account (69-71).

Thermogravimetric analysis. TGA was performed using a TGA1 (Mettler Toledo) apparatus from $30^{\circ} \mathrm{C}$ to $900^{\circ} \mathrm{C}$ with a ramp of $10^{\circ} \mathrm{C} / \mathrm{min}$ under $\mathrm{N}_{2}$ using a flow rate of $50 \mathrm{~mL} / \mathrm{min}$ and platinum pans.

Raman spectroscopy of GO, GO-DOTA samples in solution and GO-DOTA samples in urine. Samples were prepared for analysis via drop casting $20 \mu \mathrm{L}$ of $\mathrm{GO}(100 \mu \mathrm{g} / \mathrm{mL})$ dispersion onto a glass slide. Samples were left to dry for at least $2 \mathrm{~h}$ at $37^{\circ} \mathrm{C}$. For the identification of GO in the urine, $5 \mu \mathrm{L}$ of collected urine were spotted on new silica gel impregnated glass fibre sheets and then developed as described above. The developed TCL plates were then left for radioactivity decay for a month. The application points on each TCL plate were screened for GO using Raman spectroscopy. As Raman is a qualitative method of detection and urine components prove to be masking the GO signal at low concentrations, the $24 \mathrm{~h}$ collected urine was only chosen where there was the maximum amount of GO excreted for facilitating the detection of the signal.

Spectra were acquired using a micro-Raman spectrometer (Thermo Scientific, UK) using a $\lambda=633 \mathrm{~nm}$ laser at $0.4 \mathrm{~mW}$ with an exposure time of $25 \mathrm{~s}$ at a magnification of $50 \mathrm{x}$. Spectra were averaged over 5 locations and considered between $500-3500 \mathrm{~cm}^{-1}$. Post spectral processing included background correction and baseline subtraction, followed by the calculation of the $I(D) /(G)$ intensity ratio, this was completed using OriginProsoftware (version 8.5.1, Origin Lab, USA).

Animal handling procedures. Six- to eight-week-old C57BL6 mice $(18.7 \pm 2.4 \mathrm{~g})$ were obtained from Envigo (ex-Harlan) (Oxfordshire, UK), allowed to acclimatize for 1 week and were given access to food and water for 
the duration of the experiments. All procedures were carried out in accordance with the UK Home Office Code of Practice (1989) for the housing and care of animals in scientific procedures, in accordance with prior approval from the UK Home Office. All mice were injected with a single injection of either l-GO, s-GO, us-GO, l-GO-DOTA, s-GO-DOTA or us-GO-DOTA $(2.5 \mathrm{mg} / \mathrm{kg})$ or control materials such as dextrose $5 \%$ or [111 In]DOTA in $200 \mu \mathrm{L}$ by either i.v. bolus injection or infusion through the tail vein.

SPECT/CT. Mice were subjected to anaesthesia via the inhalation of $2.5 \%$ isoflurane where oxygen was used as a carrier gas set at a flow rate of $2 \mathrm{~L} / \mathrm{min}$. Each animal was then intravenously injected with the radioactive materials (either [ ${ }^{111} \mathrm{I}$ In]-GO-DOTA, [ $\left.{ }^{11} \mathrm{In}\right] \mathrm{s}$-GO-DOTA, $\left[{ }^{111} \mathrm{In}\right]$ us-GO-DOTA or [ $\left.{ }^{111} \mathrm{In}\right] \mathrm{DOTA}$ ) at a dose of 2.5 $\mathrm{mg} / \mathrm{kg}(200 \mu \mathrm{L}, 5-10 \mathrm{MBq})$. The injections were carried out by i.v. infusions through tail vein catheterisation in order to enable the dynamic imaging. At $0-1 \mathrm{~h}$ and $24 \mathrm{~h} \mathrm{SPECT/CT} \mathrm{imaging} \mathrm{was} \mathrm{carried} \mathrm{out} \mathrm{using} \mathrm{a} \mathrm{Nano-}$ Scan ${ }^{\circ}$ SPECT/CT scanner (Mediso, Hungary). SPECT images were obtained in 20 projections over 40-60 min using a 4-head scanner with $1.4 \mathrm{~mm}$ pinhole collimators for the 3D pinSPECT images and a custom made 3 $\mathrm{mm}$ single pinhole collimator for the dynamic (time laps) and static 2D images. Due to practicalities of radioisotope decay and large number of samples tested it was decided to run the fast $2 \mathrm{D}$ static scan at the mid-point $4 \mathrm{~h}$ time point rather than the full 3D pinSPECT scans that take much longer time. CT scans were taken at the end of each SPECT acquisition using a semi-circular method with full scan, 480 projections, maximum FOV, $35 \mathrm{kV}$ energy, $300 \mathrm{~ms}$ exposure time and 1-4 binning. Acquisitions were done using the Nucline v2.01 (Build 020.0000) software (Mediso, Hungary), while reconstruction of all images and fusion of SPECT with CT images was performed using the Interview ${ }^{\text {TM }}$ FUSION bulletin software (Mediso, Hungary). The images were further analysed using VivoQuant 3.0 software (Boston, US) where the SPECT images with scale bars in MBq were corrected for decay and for the slight differences in radioactivity in the injected doses between animals. Images containing scale bars in \%ID per gram were processed automatically by the VivoQuant software after providing the injected dose in MBq.

Gamma scintigraphy. For more quantitative assessment of tissue biodistribution, a cut and count study was carried out. Mice were anaesthetised by isoflurane inhalation. Each animal was injected in the tail vein with one of the radiolabelled constructs either [ ${ }^{111} \mathrm{I}$ ] $]$ l-GO-DOTA, [111 In]s-GO-DOTA, [ $\left.{ }^{111} \mathrm{In}\right]$ us-GO-DOTA or [111 In]DOTA $200 \mu \mathrm{L}$ containing $2.5 \mathrm{mg} / \mathrm{kg}$ containing approximately 5-10 MBq. Mice were sacrificed at 1, 4 and $24 \mathrm{~h}$ after injection. Blood and all major organs and tissues were collected including, heart, lungs, liver, spleen, kidneys, muscle, skin and bone. Urine and faeces were pooled and collected as well. Each sample was weighted and counted on a y-counter (Perkin Elmer, USA), together with a dilution of the injected dose with dead time limit below $60 \%$. The percentage injected dose per gram tissue was calculated, using four different mice for each time point.

Histological analysis. Lungs, liver, spleen and kidneys were extracted from mice after $24 \mathrm{~h}$ and fixed with $4 \%$ paraformaldehyde. This was followed by paraffin embedding of sections at known orientations. Sections of 5 $\mu \mathrm{m}$ were stained with $\mathrm{H}$ \& E and imaged using a 3D Histech Pannoramic 250 Flash slide scanner. Images were processed and analysed using Pannoramic Viewer (http://www.3dhistech.com/) and Fij//ImageJ software (version 1.5c; National Institutes of Health, Bethesda, MD).

Statistical analysis. Due to different sample sizes and the non-Gaussian distribution of the flake populations, the TEM size distribution data were presented using boxplots and the median, minimum and maximum values of each distribution were reported. A non-parametric test (Wilcoxon rank sum test) was performed using the statistical package in MATLAB (version R2013a, MathWorks Inc., USA), in order to determine the statistical significance of the difference between the lateral dimensions of the GO materials. Experiments were completed with an $n=3-4$ repeats and values are mean \pm standard deviation (SD). Animal experiments were completed with at least two repeats for each imaging and histological experiments and $\mathrm{n}=4$ repeats for the quantitative experiments and values are mean \pm standard error of the mean (SEM). One-way ANOVA with Tukey's posthoc test using IBM SPSS statistics 25 software were used when considering multiple comparisons for the pharmacokinetic data. 


\section{Author Contributions}

D.J. designed, planned, and led the study, performing all of the animal experiments, imaging, histopathology, radiolabelling studies and data analysis, prepared the figures and wrote the manuscript. L.N. contributed to the characterisation of GO and GO-DOTA using TEM and AFM. He performed the Raman spectroscopy experiments and helped perform the radiolabelling experiments. A.R. and N.L. prepared the initial GO and performed the physicochemical characterisation of GO and GO-DOTA samples. A.R. also helped analyse the lung histopathology data. I.V. and M. L.. performed the chemical functionalisation of GO with DOTA and performed the XPS and TGA experiments and analysis. C.M. designed and supervised the chemical reaction of GO with DOTA. A.B. provided continuous guidance in the conceptual design of the work and data interpretation. K.K. designed, planned, and discussed the findings, reviewed and edited the manuscript, and overall supervised the work. D.J., L.N., A.R., I.V., M. L., C.M., A.B. and K.K. discussed the findings and contributed to writing, reviewing, and editing the manuscript.

\section{Acknowledgments}

This work was supported by the EU 7th RTD Framework Programme, Graphene Flagship project (FP7-ICT-2013-FET-F, Project no. 604391). This work was also supported by the Agence Nationale de la Recherche (ANR) through the LabEx project Chemistry of Complex Systems (ANR-10-LABX-0026_CSC). We are very thankful to the staff at St. Mary's hospital radiopharmacy for help in delivering the radioisotopes. We are also very thankful to Mr Peter Walker at the University of Manchester histology facility for his advice and assistance in the histology analysis. We would like to acknowledge the staff from the EM Facility in the Faculty of Biology, Medicine and Health, particularly Dr Aleksandr Mironov and Ms Samantha Forbes for their expert advice and assistance. We thank $\mathrm{Dr}$ Nigel Hodson from the AFM Unit within the Biolmaging Facility in the Faculty of Biology, Medicine and Health (University of Manchester) for assistance and advice regarding the AFM instrumentation. 


\section{References}

1. K. Kostarelos, K. S. Novoselov, Graphene devices for life. Nature Nanotechnology 9, 744 (2014).

2. Y. Pan, N. G. Sahoo, L. Li, The application of graphene oxide in drug delivery. Expert Opinion on Drug Delivery 9, 1365-1376 (2012).

3. P. Avouris, F. Xia, Graphene applications in electronics and photonics. MRS Bulletin 37, 1225-1234 (2012).

4. Z. Liu, J. T. Robinson, X. Sun, H. Dai, PEGylated nanographene oxide for delivery of water-insoluble cancer drugs. Journal of Americal Chemical Society 130, 10876-10877 (2008).

5. K. Kostarelos, K. S. Novoselov, Exploring the interface of graphene and biology. Science 344, 261-263 (2014).

6. K. P. Loh, Q. Bao, G. Eda, M. Chhowalla, Graphene oxide as a chemically tunable platform for optica applications. Nature Chemistry 2, 1015-1024 (2010).

7. L. Feng, Z. Liu, Graphene in biomedicine: opportunities and challenges. Nanomedicine 6, 317-324 (2011).

8. H. Shen, L. Zhang, M. Liu, Z. Zhang, Biomedical Applications of Graphene. Theranostics 2, 283 -294 (2012).

9. I. Calizo, I. Bejenari, M. Rahman, G. Liu, A. A. Balandin, Ultraviolet Raman microscopy of single and multilayer graphene. Journal of Applied Physics 106, 043509 (2009).

10. A. C. Ferrari et al., Raman Spectrum of Graphene and Graphene Layers. Physical Review Letters 97 , $187401(2006)$

11. A. Bendali et al., Purified Neurons can Survive on Peptide-Free Graphene Layers. Advanced Healthcare Materials 10.1002/adhm.201200347, 929-933 (2013).

12. K. S. Novoselov et al., A roadmap for graphene. Nature 490, 192-200 (2012).

13. M. Zhou et al., Graphene oxide: A growth factor delivery carrier to enhance chondrogenic differentiation of human mesenchymal stem cells in 3D hydrogels. Acta Biomaterialia 96, 271-280 (2019)

14. N. A. Kotov et al., Nanomaterials for Neural Interfaces. Advanced Materials 21, 3970-4004 (2009).

15. S. K. Seidlits, J. Y. Lee, C. E. Schmidt, Nanostructured scaffolds for neural applications. Nanomedicine (London, England) 3, 183-199 (2008).

16. K. Yang et al., In Vivo Pharmacokinetics, Long-Term Biodistribution, and Toxicology of PEGylated Graphene in Mice. ACS Nano 5, 516-522 (2011).

17. J. H. Liu et al., Effect of size and dose on the biodistribution of graphene oxide in mice. Nanomedicine (London, England) 7, 1801-1812 (2012).

18. L. Zhan et al., Biodistribution of co-exposure to multi-walled carbon nanotubes and graphene oxide nanoplatelets radiotracers. J Nanopart Res 13, 2939-2947 (2011).

19. G. Qu et al., The ex vivo and in vivo biological performances of graphene oxide and the impact of surfactant on graphene oxide's biocompatibility. Journal of Environmental Sciences 25, 873-881 (2013).

20. K. Yang et al., In vivo biodistribution and toxicology of functionalized nano-graphene oxide in mice after oral and intraperitoneal administration. Biomaterials 34, 2787-2795 (2013).

21. L. Yan et al., Can graphene oxide cause damage to eyesight? Chemical Research Toxicology 25, 12651270 (2012).

22. C. M. Girish, A. Sasidharan, G. S. Gowd, S. Nair, M. Koyakutty, Confocal Raman Imaging Study Showing Macrophage Mediated Biodegradation of Graphene In Vivo. Advanced Healthcare Materials 2, 14891500 (2013).

23. K. Wang et al., Biocompatibility of Graphene Oxide. Nanoscale Research Letters 6, 8-8 (2011).

24. X. Zhang et al., Distribution and biocompatibility studies of graphene oxide in mice after intravenous administration. Carbon 49, 986-995 (2011).

25. S. Zhang, K. Yang, L. Feng, Z. Liu, In vitro and in vivo behaviors of dextran functionalized graphene. Carbon 49, 4040-4049 (2011).

26. K. Yang et al., Graphene in Mice: Ultrahigh In Vivo Tumor Uptake and Efficient Photothermal Therapy. Nano Letters 10, 3318-3323 (2010).

27. D. A. Jasim et al., Thickness of functionalized graphene oxide sheets plays critical role in tissue accumulation and urinary excretion: A pilot PET/CT study. Applied Materials Today 4, 24-30 (2016).

28. D. A. Jasim, C. Menard-Moyon, D. Begin, A. Bianco, K. Kostarelos, Tissue distribution and urinary excretion of intravenously administered chemically functionalized graphene oxide sheets. Chemical Science 6, 3952-3964 (2015).

29. D. A. Jasim et al., The Effects of Extensive Glomerular Filtration of Thin Graphene Oxide Sheets on Kidney Physiology. ACS Nano 10, 10753-10767 (2016).

30. A. Bianco, Graphene: Safe or Toxic? The Two Faces of the Medal. Angewandte Chemie International Edition 52, 4986-4997 (2013).

31. P. Wick et al., Classification Framework for Graphene-Based Materials. Angewandte Chemie International Edition 10.1002/anie.201403335, 2-7 (2014).

32. R. P. Heaney, Factors influencing the measurement of bioavailability, taking calcium as a model. Journal of Nutrition 131, 1344S-1348S (2001).

33. B. Cornelissen et al., Nanographene oxide-based radioimmunoconstructs for in vivo targeting and SPECT imaging of HER2-positive tumors. Biomaterials 34, 1146-1154 (2013). 
34. H. Hong et al., In Vivo Targeting and Imaging of Tumor Vasculature with Radiolabeled, AntibodyConjugated Nanographene. ACS Nano 6, 2361-2370 (2012).

35. D. A. Jasim, N. Lozano, K. Kostarelos, Synthesis of few-layered, high-purity graphene oxide sheets from different graphite sources for biology. 2D Materials 3, 014006 (2016).

36. S. P. Mukherjee et al., Detection of Endotoxin Contamination of Graphene Based Materials Using the TNF- $\alpha$ Expression Test and Guidelines for Endotoxin-Free Graphene Oxide Production. PLOS ONE 11, e0166816 (2016).

37. A. F. Rodrigues et al., A blueprint for the synthesis and characterisation of thin graphene oxide with controlled lateral dimensions for biomedicine. 2D Materials 5, 035020 (2018).

38. S. Bhattacharjee, DLS and zeta potential - What they are and what they are not? Journal of controlled release : official journal of the Controlled Release Society 235, 337-351 (2016).

39. J. L. Li et al., Oxygen-driven unzipping of graphitic materials. Phys Rev Lett 96, 176101 (2006).

40. T. Sun, S. Fabris, Mechanisms for oxidative unzipping and cutting of graphene. Nano Lett 12, 17-21 (2012).

41. W. Zhang et al., Unraveling stress-induced toxicity properties of graphene oxide and the underlying mechanism. Advanced materials (Deerfield Beach, Fla.) 24, 5391-5397 (2012).

42. A. M. Dimiev, L. B. Alemany, J. M. Tour, Graphene oxide. Origin of acidity, its instability in water, and a new dynamic structural model. ACS Nano 7, 576-588 (2013).

43. S. Eigler, C. Dotzer, A. Hirsch, M. Enzelberger, P. Müller, Formation and Decomposition of CO2 Intercalated Graphene Oxide. Chemistry of Materials 24, 1276-1282 (2012).

44. I. A. Vacchi, C. Spinato, J. Raya, A. Bianco, C. Menard-Moyon, Chemical reactivity of graphene oxide towards amines elucidated by solid-state NMR. Nanoscale 8, 13714-13721 (2016).

45. S. Stankovich et al., Synthesis of graphene-based nanosheets via chemical reduction of exfoliated graphite oxide. Carbon 45, 1558-1565 (2007).

46. J. I. Paredes, S. Villar-Rodil, A. Martínez-Alonso, J. M. Tascón, Graphene oxide dispersions in organic solvents. Langmuir: the ACS journal of surfaces and colloids 24, 10560-10564 (2008).

47. I. A. Vacchi, S. Guo, J. Raya, A. Bianco, C. Ménard-Moyon, Strategies for the Controlled Covalent Double Functionalization of Graphene Oxide. Chemistry - A European Journal 26, 6591-6598 (2020).

48. M. Orecchioni et al., Molecular and Genomic Impact of Large and Small Lateral Dimension Graphene Oxide Sheets on Human Immune Cells from Healthy Donors. Advanced Healthcare Materials 5, 276-287 (2016).

49. A. F. Rodrigues et al., Size-Dependent Pulmonary Impact of Thin Graphene Oxide Sheets in Mice: Toward Safe-by-Design. Advanced Science n/a, 1903200.

50. L. Newman et al., Nose-to-Brain Translocation and Cerebral Biodegradation of Thin Graphene Oxide Nanosheets. Cell Reports Physical Science 1, 100176 (2020).

51. W. Zhang et al., Unraveling Stress-Induced Toxicity Properties of Graphene Oxide and the Underlying Mechanism. Advanced Materials 24, 5391-5397 (2012).

52. Y. Li et al., Surface Coating-Dependent Cytotoxicity and Degradation of Graphene Derivatives: Towards the Design of Non-Toxic, Degradable Nano-Graphene. Small 10.1002/smll.201303234, 1544-1554 (2013).

53. I. Jung et al., Reduction Kinetics of Graphene Oxide Determined by Electrical Transport Measurements and Temperature Programmed Desorption. The Journal of Physical Chemistry C 113, 18480-18486 (2009).

54. F. Alexis, E. Pridgen, L. K. Molnar, O. C. Farokhzad, Factors Affecting the Clearance and Biodistribution of Polymeric Nanoparticles. Molecular Pharmaceutics 5, 505-515 (2008).

55. E. Blanco, H. Shen, M. Ferrari, Principles of nanoparticle design for overcoming biological barriers to drug delivery. Nature Biotechnology 33, 941-951 (2015).

56. K. Wang et al., Biocompatibility of graphene oxide. Nanoscale Res Lett 6, 1-8 (2011).

57. S. K. Singh et al., Thrombus Inducing Property of Atomically Thin Graphene Oxide Sheets. ACS Nano 5 , 4987-4996 (2011).

58. M. Geiser, Update on macrophage clearance of inhaled micro- and nanoparticles. Journal of Aerosol Medicine Pulmonary Drug Delivery 23, 207-217 (2010).

59. K. Yang et al., The influence of surface chemistry and size of nanoscale graphene oxide on photothermal therapy of cancer using ultra-low laser power. Biomaterials 33, 2206-2214 (2012).

60. H. Hong et al., In vivo targeting and positron emission tomography imaging of tumor vasculature with (66)Ga-labeled nano-graphene. Biomaterials 33, 4147-4156 (2012).

61. M. Zhang et al., Radiolabeling, whole-body single photon emission computed tomography/computed tomography imaging, and pharmacokinetics of carbon nanohorns in mice. International Journal of Nanomedicine 11, 3317-3330 (2016).

62. S. Liang et al., In vivo pharmacokinetics, transfer and clearance study of graphene oxide by La/Ce dual elemental labelling method. Nanolmpact 17, 100213 (2020).

63. B. T. Kurien, N. E. Everds, R. H. Scofield, Experimental animal urine collection: a review. Lab Animals 38, 333-361 (2004).

64. B. Li et al., Influence of polyethylene glycol coating on biodistribution and toxicity of nanoscale graphene oxide in mice after intravenous injection. Intenational Journal of Nanomedicine 9, 4697-4707 (2014).

65. L. Newman et al., Splenic Capture and In Vivo Intracellular Biodegradation of Biological-Grade Graphene Oxide Sheets. ACS Nano 14, 10168-10186 (2020). 
1 66. M. C. Duch et al., Minimizing Oxidation and Stable Nanoscale Dispersion Improves the Biocompatibility of Graphene in the Lung. Nano Letters 11, 5201-5207 (2011).

67. L. Ma-Hock et al., Comparative inhalation toxicity of multi-wall carbon nanotubes, graphene, graphite nanoplatelets and low surface carbon black. Particle and Fibre Toxicology 10, 23 (2013).

68. S. Macholl et al., High-throughput high-volume nuclear imaging for preclinical in vivo compound screening(§). EJNMMI Research 7, 33 (2017).

69. A. Ganguly, S. Sharma, P. Papakonstantinou, J. Hamilton, Probing the Thermal Deoxygenation of Graphene Oxide Using High-Resolution In Situ X-ray-Based Spectroscopies. The Journal of Physical Chemistry C 115, 17009-17019 (2011).

70. L.-N. Zhou, X.-T. Zhang, W.-J. Shen, S.-G. Sun, Y.-J. Li, Monolayer of close-packed Pt nanocrystals on a reduced graphene oxide (RGO) nanosheet and its enhanced catalytic performance towards methano electrooxidation. RSC Advances 5, 46017-46025 (2015).

71. C. Botas et al., Tailored graphene materials by chemical reduction of graphene oxides of different atomic structure. RSC Advances 2, 9643-9650 (2012). 


\section{Figure Legends}

Scheme 1. Synthesis of GO-DOTA. For the sake of clarity, electrostatic interactions between the protonated amine in $\mathrm{NH}_{2}-\mathrm{PEG}_{4}-\mathrm{DOTA}$ and the carboxylate groups at the edges of $\mathrm{GO}$ or in the $\mathrm{PEG}_{4}$-DOTA chain linked to $\mathrm{GO}$ are not shown.

Figure 1. Physicochemical characterisation of I-GO, s-GO and us-GO before and after DOTA functionalisation. A) Dynamic light scattering (DLS) size distributions; B) Electrophoretic mobility ( $\zeta$ potential) mean surface charge data. Morphological and structural characterisation data is shown, using C) TEM and D) AFM.

Figure 2: Radiolabelling efficiency and stability. A) Efficiency of radiolabelling of the three types of GO ([111 In]l-GO-DOTA, [ $\left.{ }^{111} \mathrm{In}\right] \mathrm{s}-\mathrm{GO}-D O T A$ and $\left[{ }^{111} \mathrm{In}\right]$ us-GO-DOTA) compared to control [111 In]DOTA after the radiolabelling reaction; values indicate the average of three independent labelling repeats. B) Radiolabelling purity of the three samples after centrifugation and before administration in the animals. C) Stability of radiolabelling in PBS and $50 \%$ serum up to 7 days

Figure 3: Biodistribution of the three types of GO ([ $\left.{ }^{111} \mathrm{In}\right] \mathrm{I}-\mathrm{GO}-\mathrm{DOTA},\left[{ }^{111} \mathrm{In}\right] \mathrm{s}-\mathrm{GO}-\mathrm{DOTA}$ and [111 In]us-GO-DOTA) compared to [ $\left.{ }^{111} \mathrm{In}\right]$ DOTA control at $1 \mathrm{~h}$ and $24 \mathrm{~h}$. A) Blood profile up to $24 \mathrm{~h}$. B) SPECT/CT images expressed as \% of injected dose per gram of tissues (doses were decay corrected at the second time point). From left to right (whole body maximum intensity projections (MIP), sagittal, coronal and transverse views). Interactive 3D images of the MIPs are available online. C) Organ distribution of the three materials as determined by $\mathrm{Y}$-counting. Four animals per group were used for $A$ and $C$, while two animals were used for the imaging in $B$.

Figure 4: Excretion profile of GO ([111In]I-GO-DOTA, [111/n]s-GO-DOTA and [ $\left.{ }^{111} \mathrm{In}\right]$ us-GO-DOTA compared to control [111In]DOTA). A) Urinary excretion profile of the three materials at different time points $B$ ) detection of the graphene material in the urine 24 hours post administration of mice as demonstrated by radio-TLC and corroborative Raman spectroscopy, scale bars are $20 \mu \mathrm{m}$. C) Faecal excretion of the three materials compared to the control.

Figure 5. Effect of I-GO-DOTA, s-GO-DOTA, us-GO-DOTA on lung compared to control $5 \%$ dextrose. Haematoxylin and eosin stained lung sections ( $5 \mu \mathrm{m}$ thick) after injection of I-GO-DOTA, S-GO-DOTA, us-GO-DOTA and 5\% dextrose (negative control) after $24 \mathrm{~h}$ of two different mice for each material. Scale bars for the images on the left are $100 \mu \mathrm{m}$ while those on the right are $20 \mu \mathrm{m}$. 


\section{Figures}

2

4

5

6

10

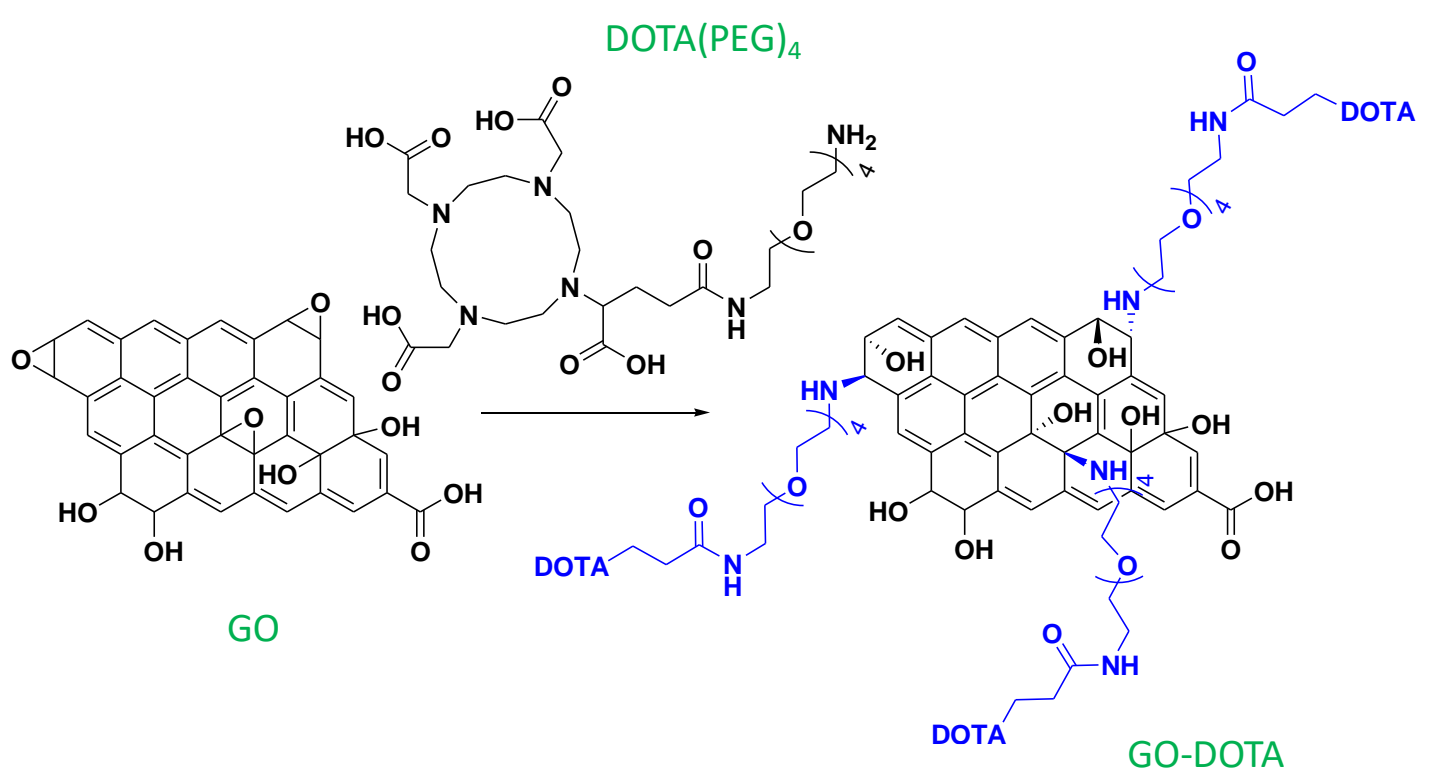

Scheme 1. Synthesis of GO-DOTA. For the sake of clarity, electrostatic interactions between the protonated amine in $\mathrm{NH}_{2}-\mathrm{PEG}_{4}$-DOTA and the carboxylate groups at the edges of $\mathrm{GO}$ or in the $\mathrm{PEG}_{4}$-DOTA chain linked to $\mathrm{GO}$ are not shown.

12

13 


\section{$1 \quad$ Figure 1}

2

3
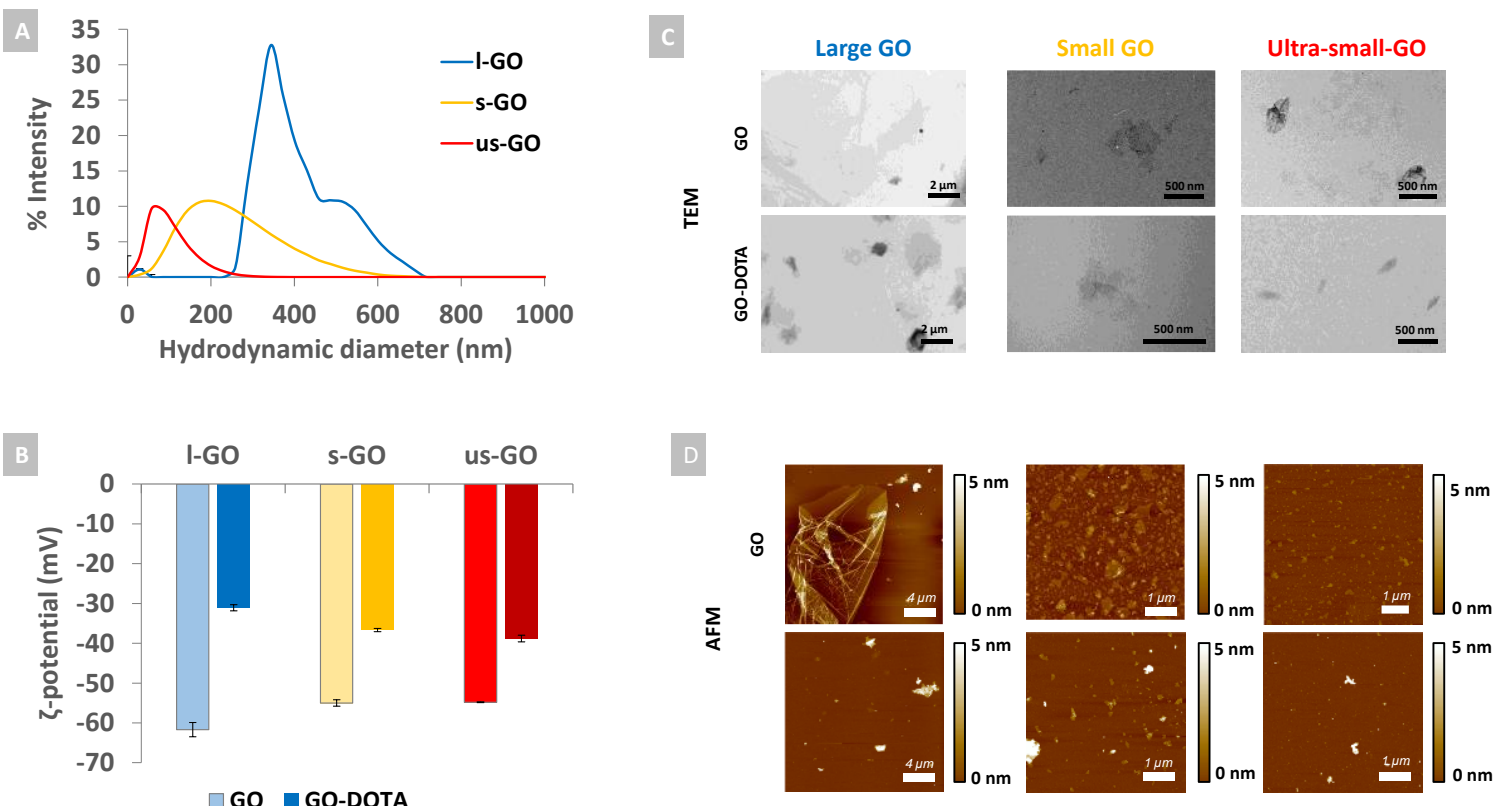

Figure 1. Physicochemical characterisation of I-GO, s-GO and us-GO before and after DOTA functionalisation. A) Dynamic light scattering (DLS) size distributions; B) Electrophoretic mobility

8 ( $\zeta$ potential) mean surface charge data. Morphological and structural characterisation data is 9 shown, using C) TEM and D) AFM. 


\section{$1 \quad$ Figure 2}

2

A

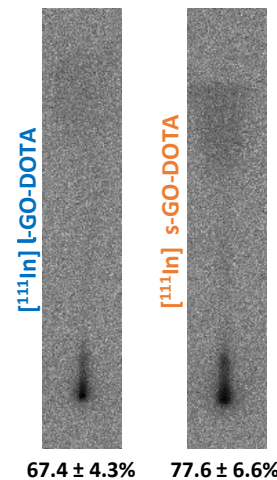

$67.4 \pm 4.3 \% \quad 77.6 \pm 6.6 \%$

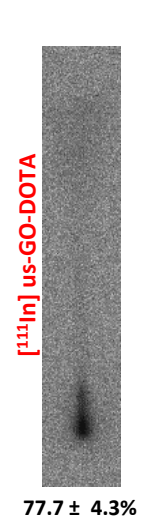

$86.3 \pm 7.8 \%$

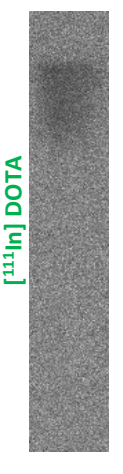

B

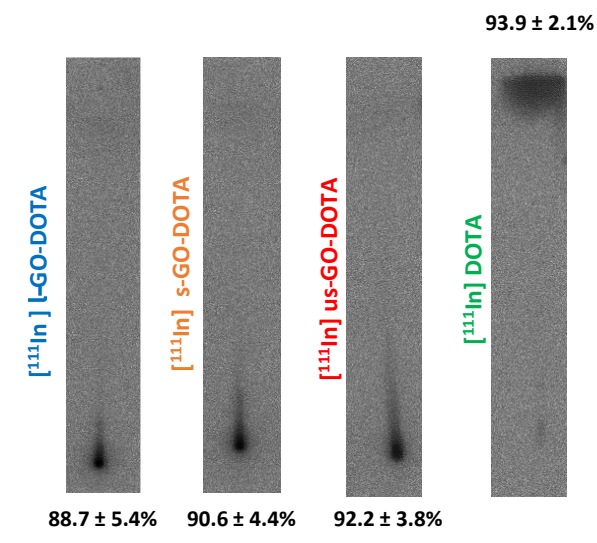

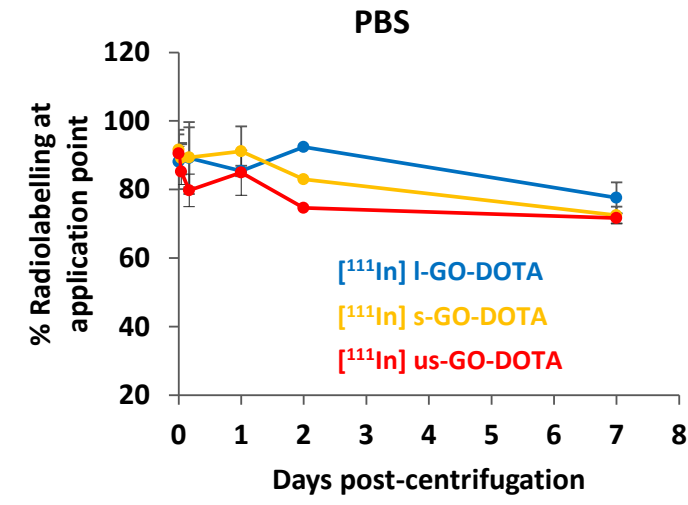

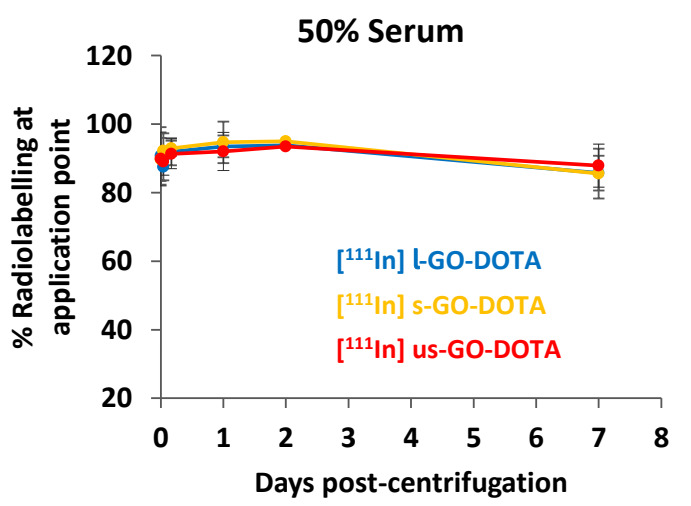

Figure 2: Radiolabelling efficiency and stability. A) Efficiency of radiolabelling of the three types of GO ([111 In] I-GO-DOTA, [ $\left.{ }^{111} \mathrm{In}\right] \mathrm{s}$ s-GO-DOTA and [ $\left.{ }^{111} \mathrm{In}\right]$ us-GO-DOTA) compared to control $\left[{ }^{111} \mathrm{In}\right] \mathrm{DOT} A$ after the radiolabelling reaction; values indicate the average of three independent labelling repeats. B) Radiolabelling purity of the three samples after centrifugation and before administration in the animals. C) Stability of radiolabelling in PBS and 50\% serum up to 7 days. 


\section{$1 \quad$ Figure 3}

2

3

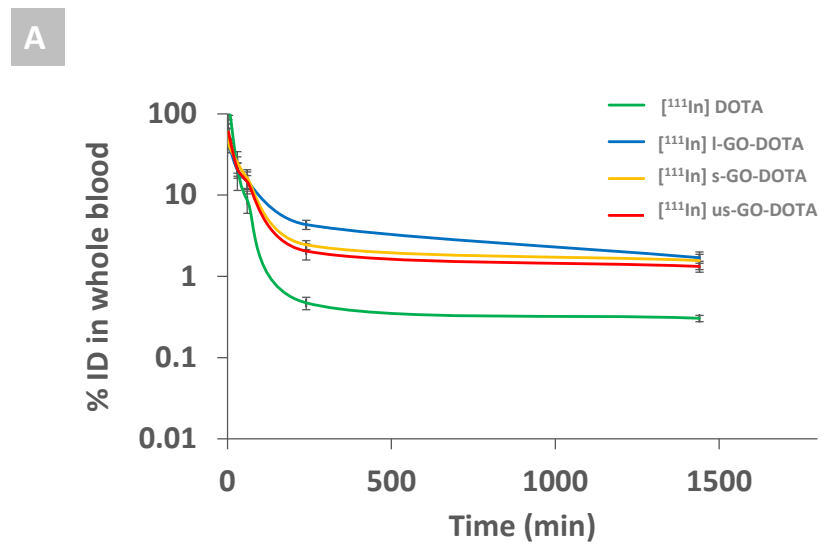

B

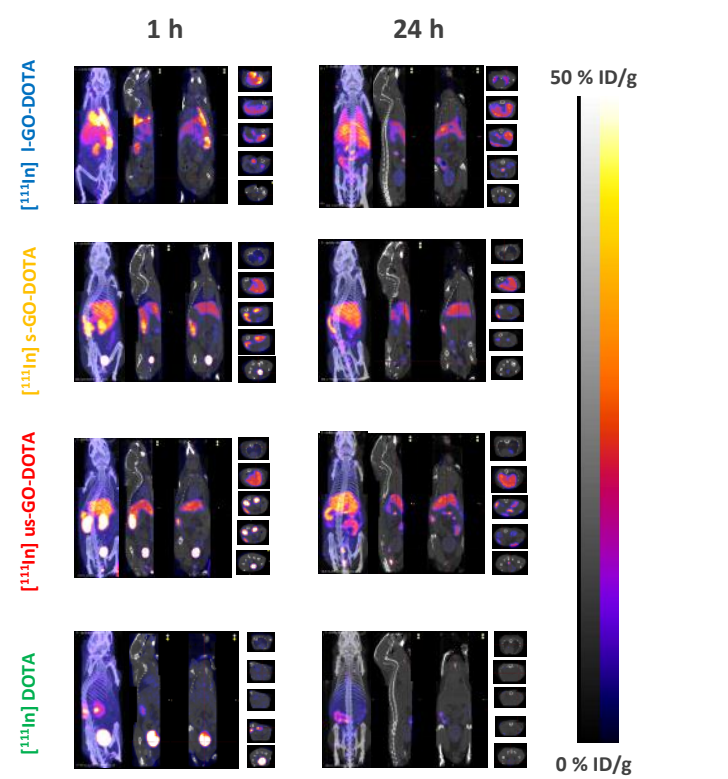

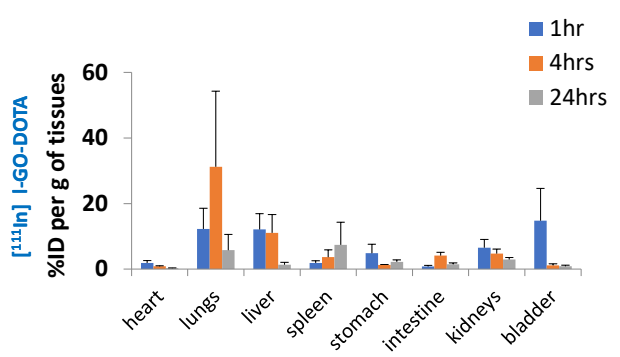
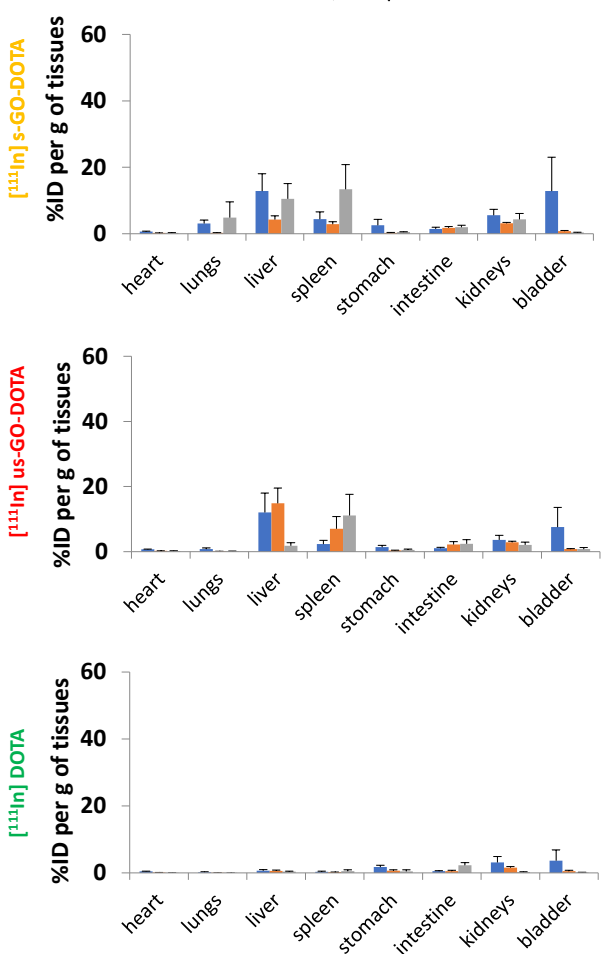

Figure 3: Biodistribution of the three types of GO ([111In] I-GO-DOTA, $\left[{ }^{111} \mathrm{In}\right] \mathrm{s}$-GO-DOTA and [111/n] us-GO-DOTA) compared to [111In]DOTA control at $1 \mathrm{~h}$ and $24 \mathrm{~h}$. A) Blood profile up to 24h. B) SPECT/CT images expressed as \% of injected dose per gram of tissues (doses were decay corrected at the second time point). From left to right (whole body maximum intensity projections (MIP), sagittal, coronal and transverse views). Interactive 3D images of the MIPs are available online. C) Organ distribution of the three materials as determined by $\mathrm{Y}$-counting. Four animals per group were used for $A$ and $C$, while two animals were used for the imaging in (B). 
3

\section{$4 \quad$ Figure 4}

5
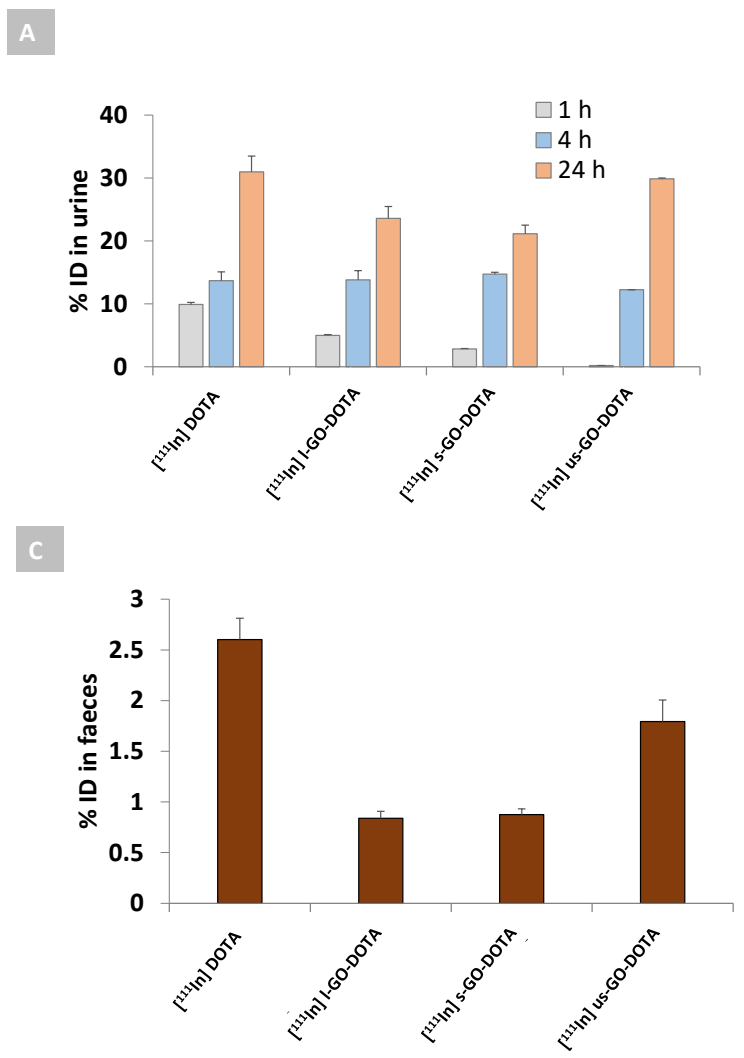

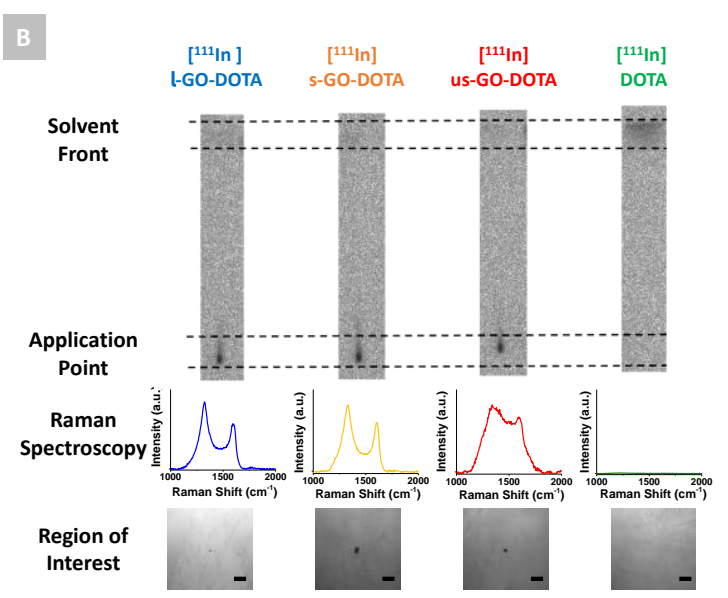

6

Figure 4: Excretion profile of I-, s- and us-GO ([111/I]l-GO-DOTA, [111/n]s-GO-DOTA and ['11In]us-GO-DOTA) compared to control [ ${ }^{111}$ In]DOTA. A) Urinary excretion profile of the three materials at different time points; B) detection of the graphene material in the urine 24 hours post administration of mice as demonstrated by radio-TLC and corroborative Raman spectroscopy (scale bars are $20 \mu \mathrm{m}$ ); $\mathbf{C}$ ) faecal excretion of the three materials compared to the control. 
Figure 5
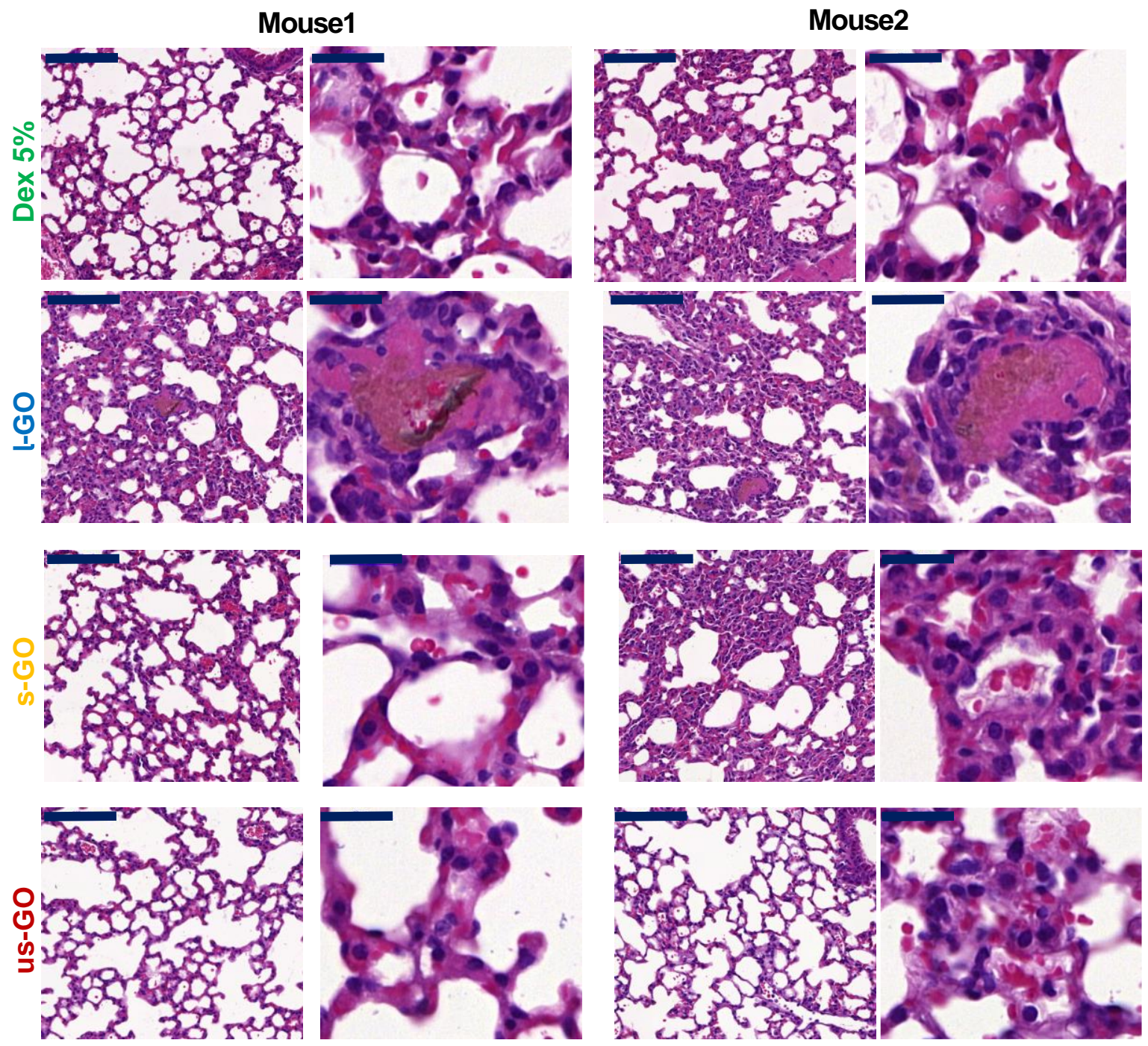

Figure 5. Effect of non-radiolabelled I-GO-DOTA, s-GO-DOTA, us-GO-DOTA on lung compared to vehicle alone ( $5 \%$ dextrose). Haematoxylin and eosin stained lung sections $(5 \mu \mathrm{m}$ thick) after injection of l-GO-DOTA, s-GO-DOTA, us-GO-DOTA and 5\% dextrose (negative control) after $24 \mathrm{~h}$ of two different mice for each material. Two magnifications were used, with scale bars on the left in each panel showing $100 \mu \mathrm{m}$, while those on the right are indicating $20 \mu \mathrm{m}$. 


\section{1 \\ Supporting Information}

3

4

5
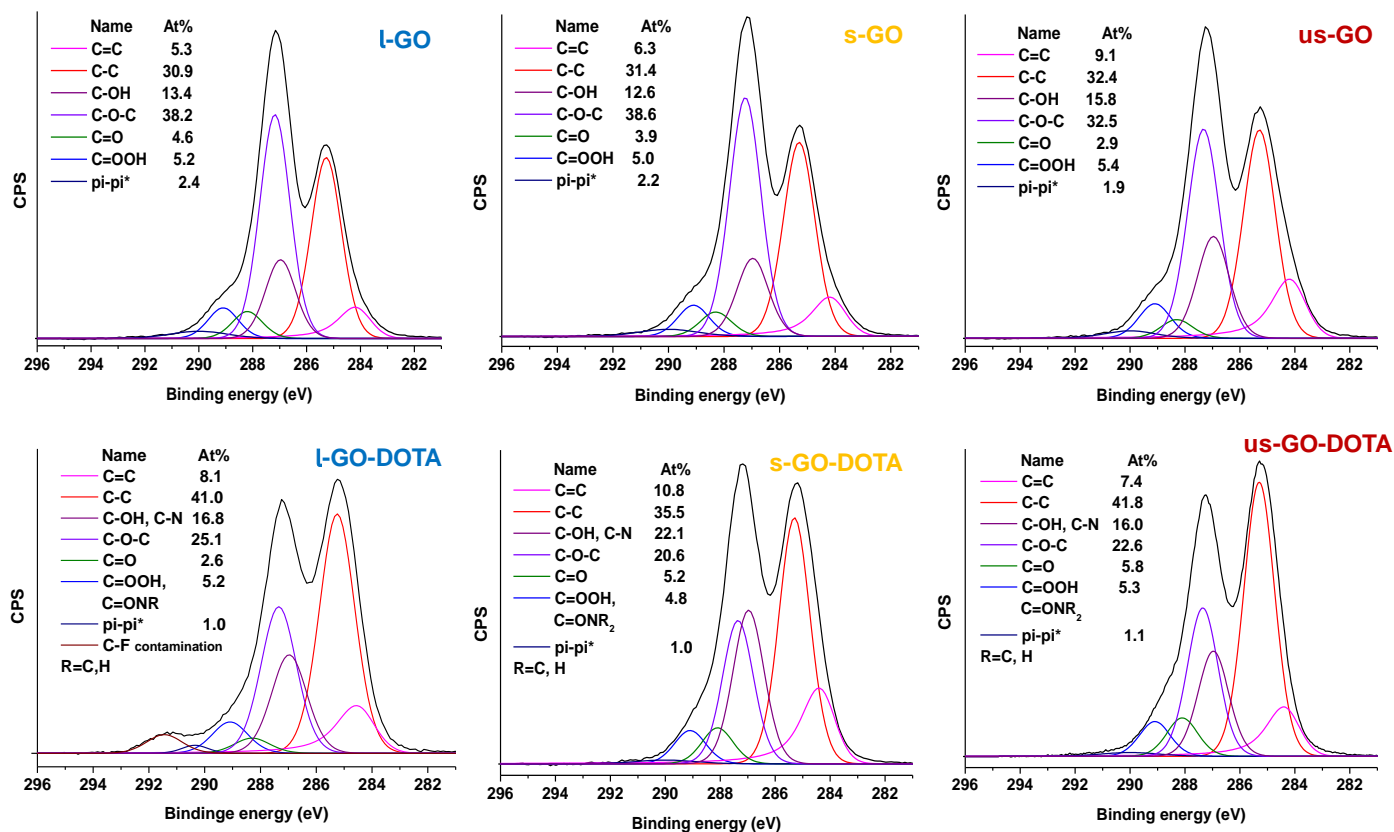

Figure S1: C1s XPS spectra showing carbon high resolution spectra of all three GO materials with their DOTA functionalized counterparts. 
1

2

3

4

5

6
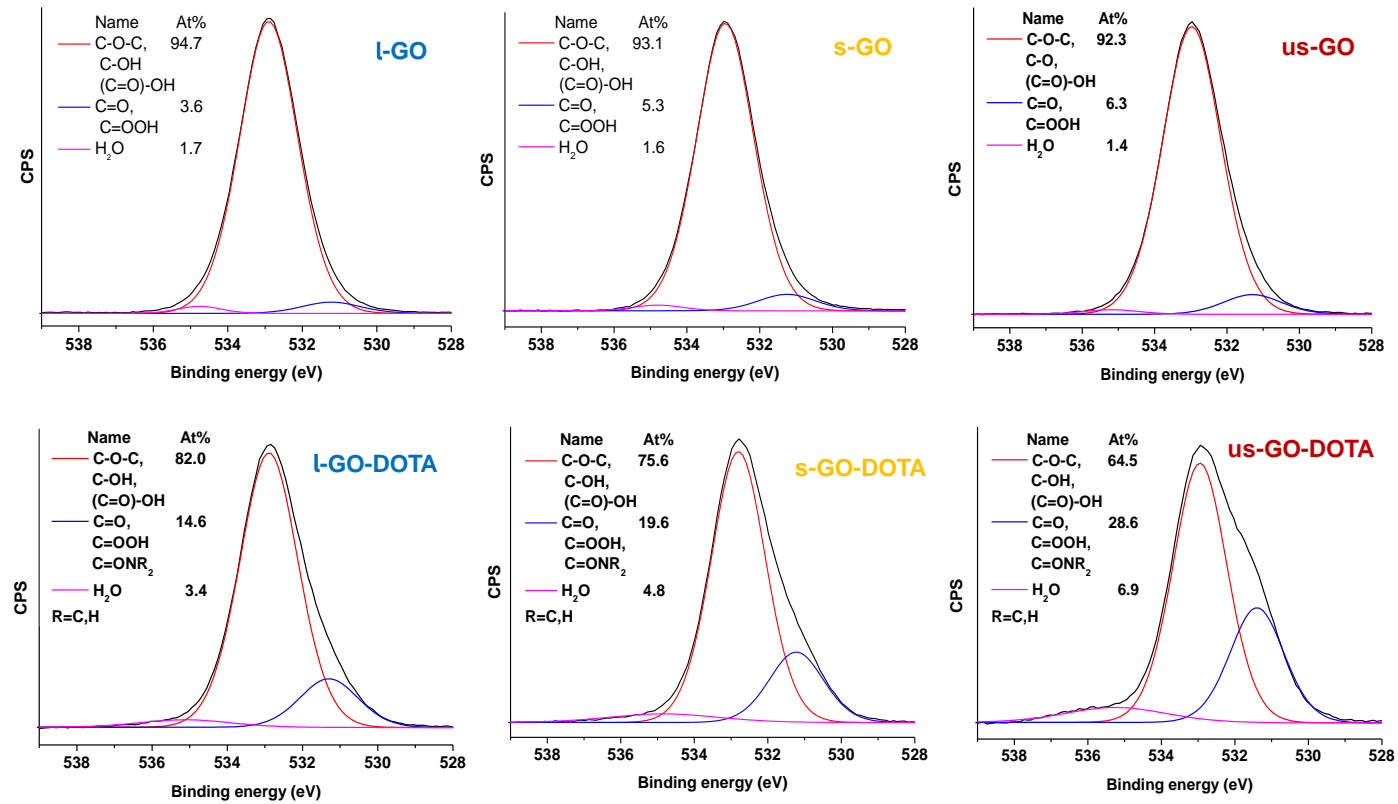

Figure S2: O1s XPS spectra showing oxygen high resolution spectra of all three GO materials with their DOTA functionalized counterparts. 

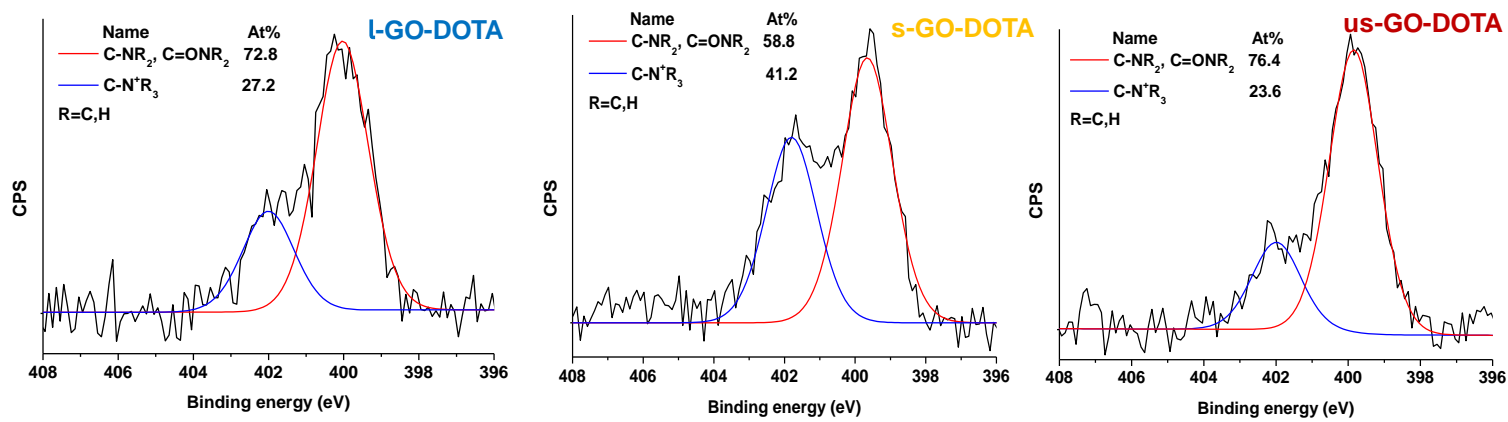

Figure S3: N1s XPS spectra showing nitrogen high resolution spectra of all three GO starting materials with their DOTA- functionalized counterparts. 


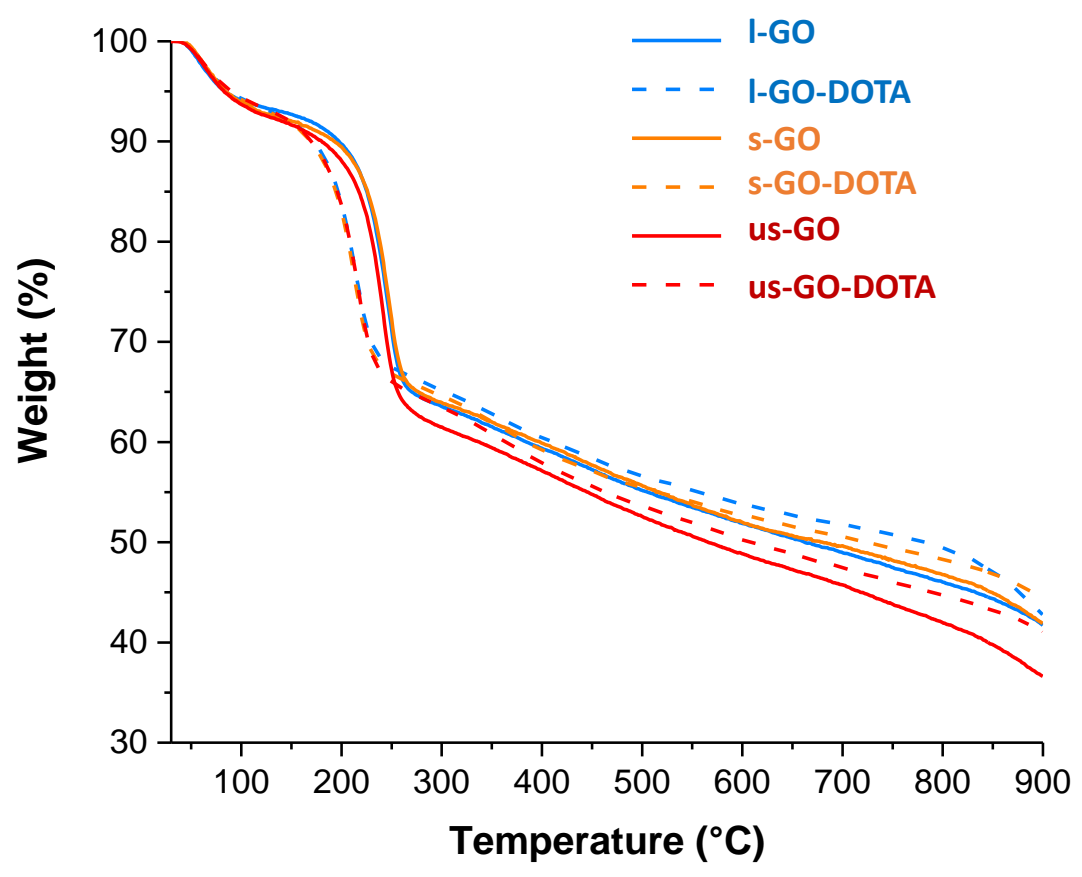

2

3 Figure S4: TGA of the three starting GO materials and their functionalized conjugates.

4 

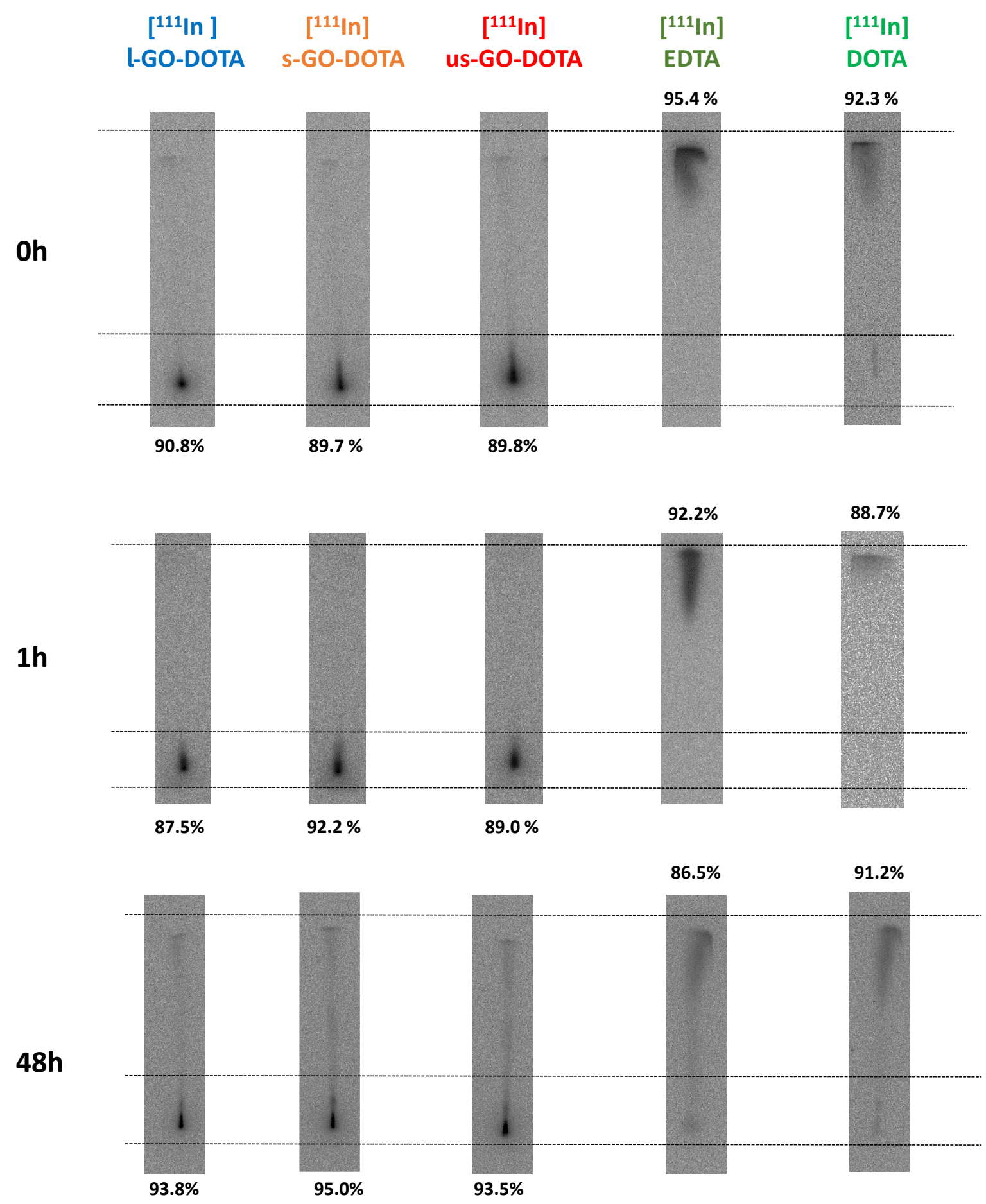

3 Figure S5: Radiolabeling stability in serum. TLC strips of the samples $\left[{ }^{111} \mathrm{In}\right] \mathrm{l}-\mathrm{GO}-\mathrm{DOTA},\left[{ }^{111} \mathrm{In}\right]$ 4 GO-DOTA, [ $\left.{ }^{111} \mathrm{In}\right]$ us-GO-DOTA [ $\left.{ }^{111} \mathrm{In}\right]$ EDTA and [ $\left.{ }^{111} \mathrm{In}\right] \mathrm{DOTA}$ control in $50 \%$ serum at different time 5 points.

6

7 

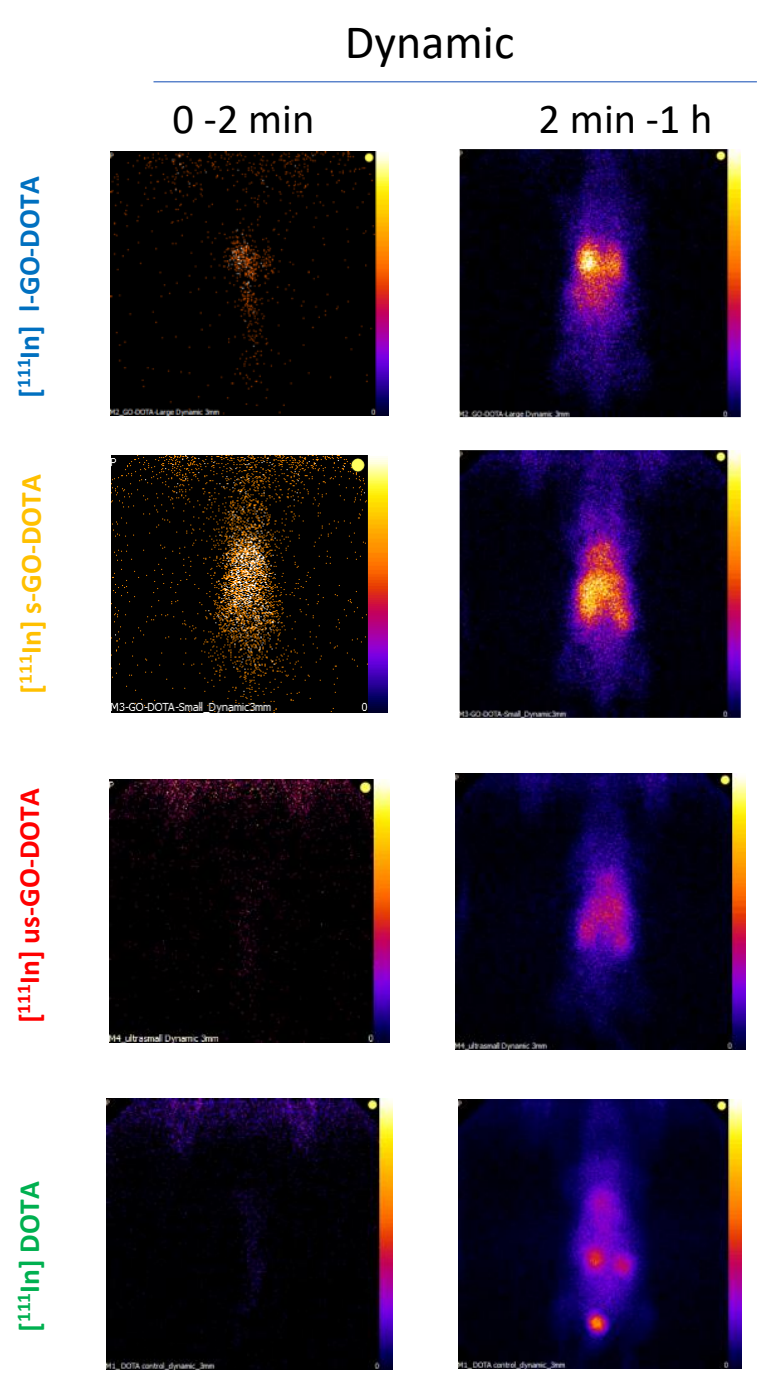

Static Planar

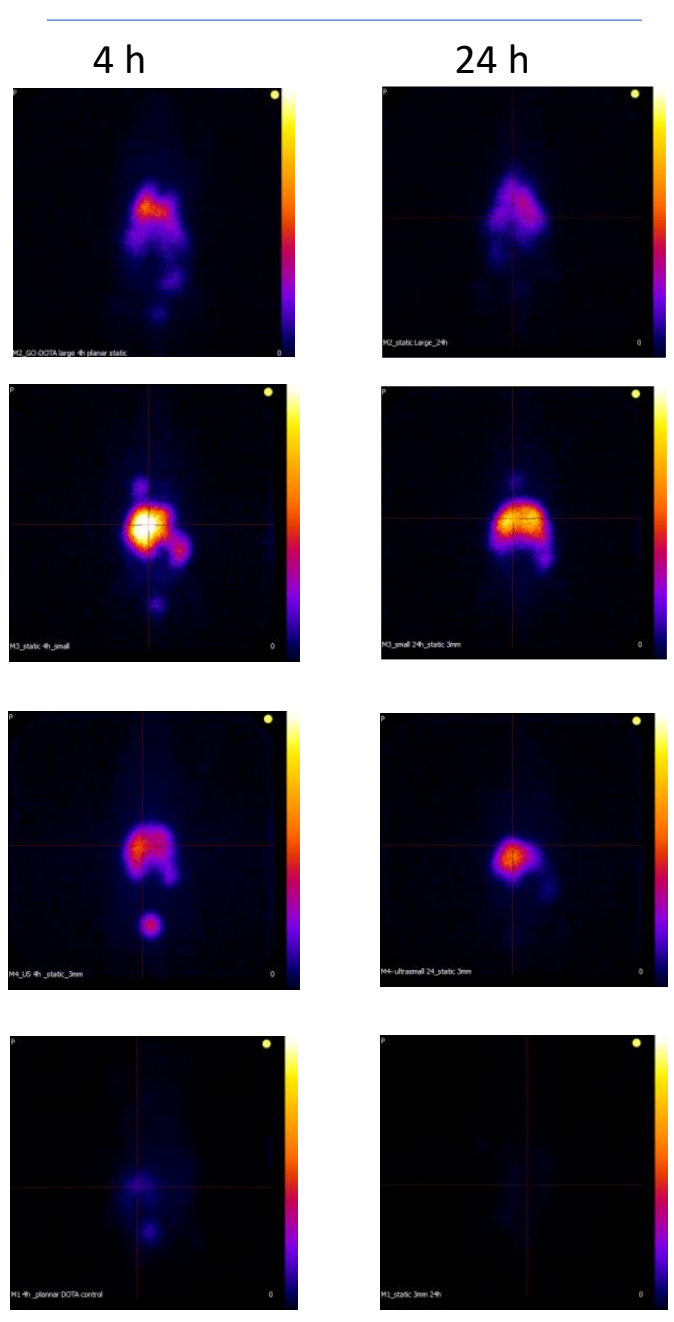

Figure S6: Planar SPECT data showing the biodistribution of the three types of GO $\left(\left[{ }^{111} \mathrm{In}\right] \mathrm{GO}\right.$ DOTA, [ $\left.{ }^{111} \mathrm{In}\right] \mathrm{s}-\mathrm{GO}-\mathrm{DOTA}$ and $\left[{ }^{111} \mathrm{In}\right]$ us-GO-DOTA) compared to control $\left[{ }^{111} \mathrm{In}\right] \mathrm{DOTA}$. A) The first two columns show the dynamic videos of the injection phase $(2 \mathrm{~min})$ and $1 \mathrm{~h}$ dynamic profile, while the two columns on the right are the planer static images at $4 \mathrm{~h}$ and $24 \mathrm{~h}$. Dynamic videos are available online. 

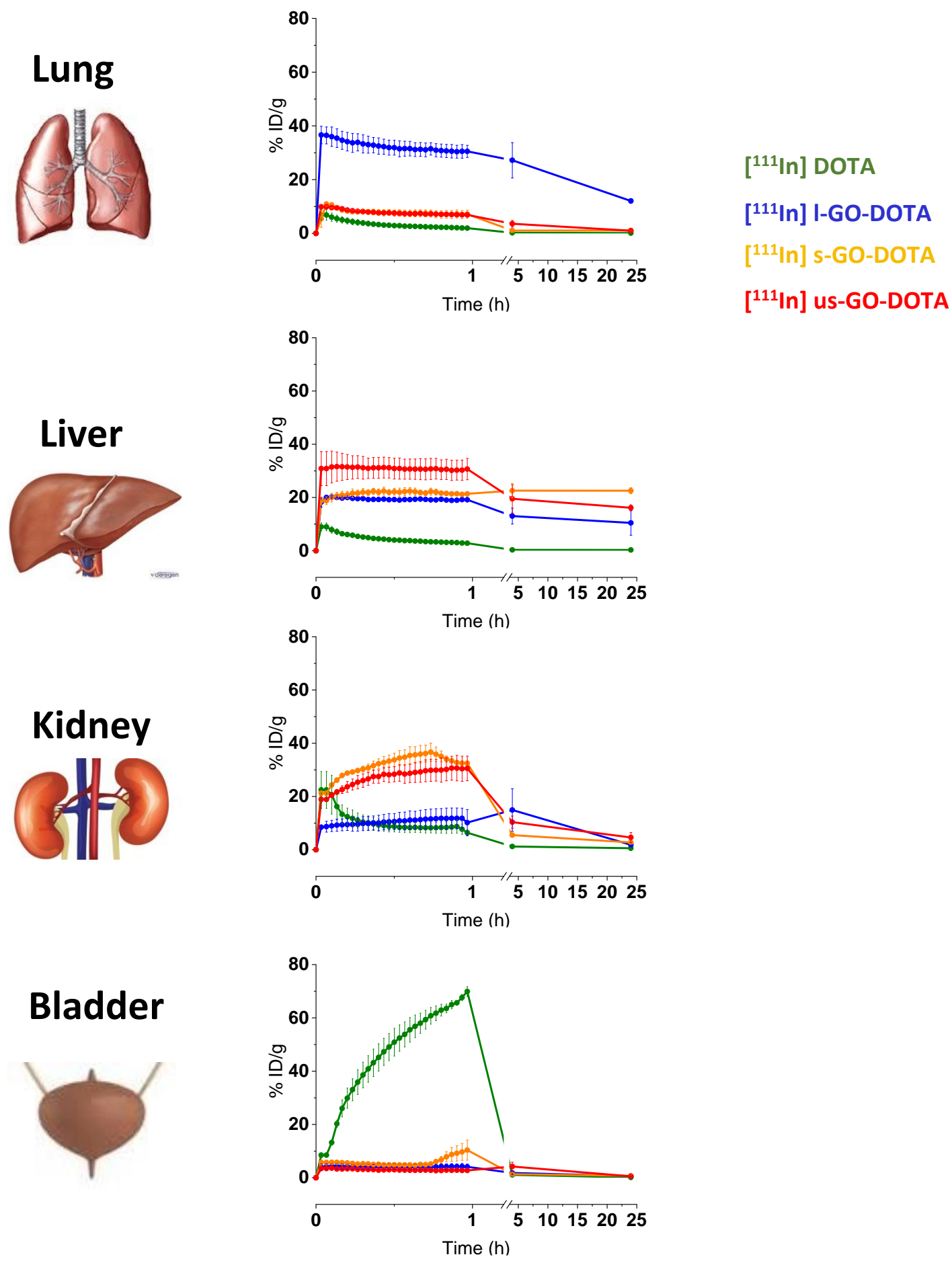

Figure S7: Organ distribution of the three types of GO ([111 In]l-GO-DOTA, $\left[{ }^{111} \mathrm{In}\right] \mathrm{s}$-GO-DOTA and [111In]us-GO-DOTA) compared to control [ $\left.{ }^{111} \mathrm{In}\right]$ DOTA demonstrated by the time activity curve of the corresponding planar SPECT data $(n=2)$. 

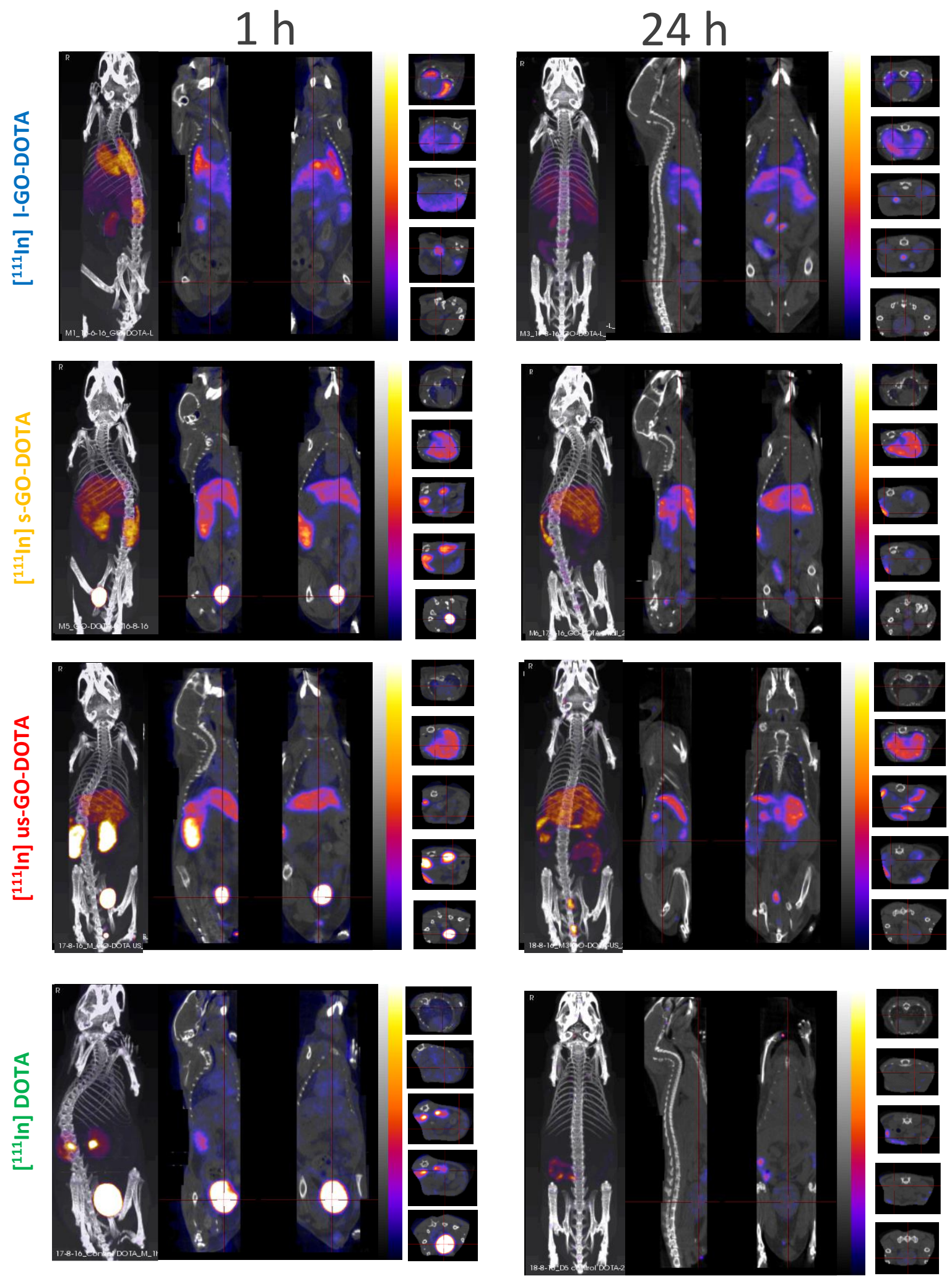

Figure S8: Biodistribution of the three types of GO ([111 In]l-GO-DOTA, [ $\left.{ }^{111} \mathrm{In}\right] \mathrm{s}-\mathrm{GO}-D O T A$ and [111In]us-GO-DOTA) compared to control [111 In]DOTA at $1 \mathrm{~h}$ and $24 \mathrm{~h}$ in a first batch of mice. From left to right (whole body maximum intensity projections (MIP), sagittal, coronal and transverse views). The data here are normalized MBq for decay and dose differences between mice. Interactive 3D images (of MIPs) are available online. 
$1 \mathrm{~h}$
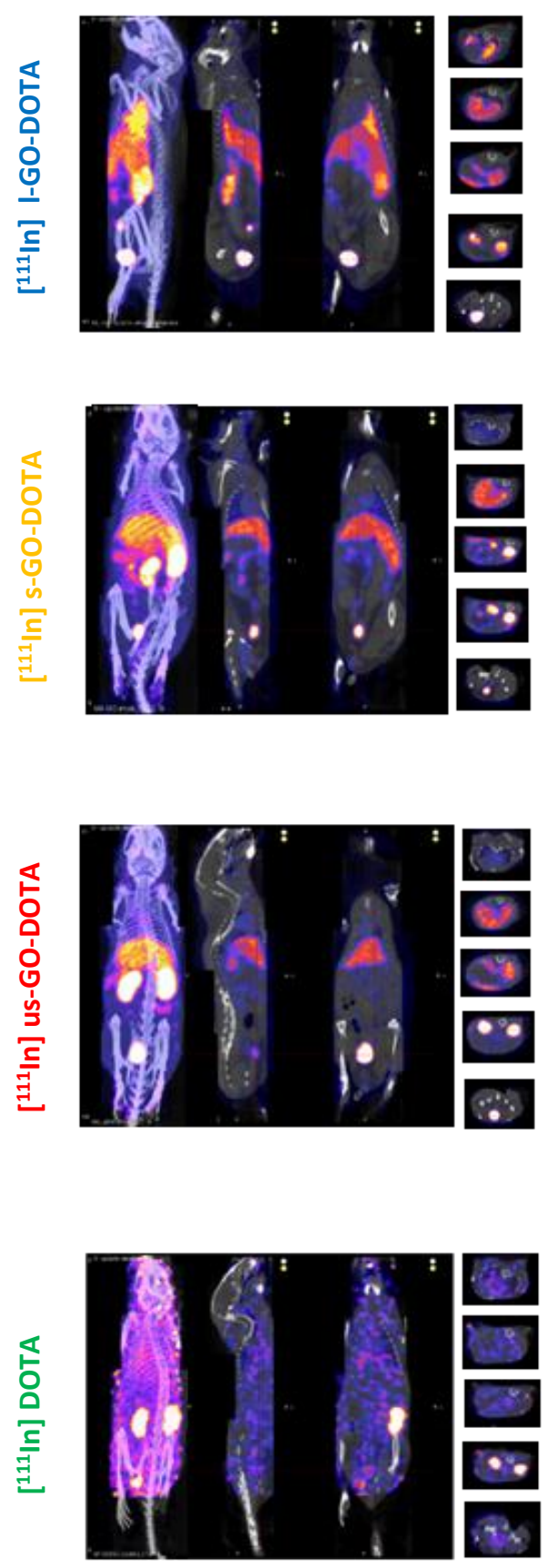

$24 \mathrm{~h}$
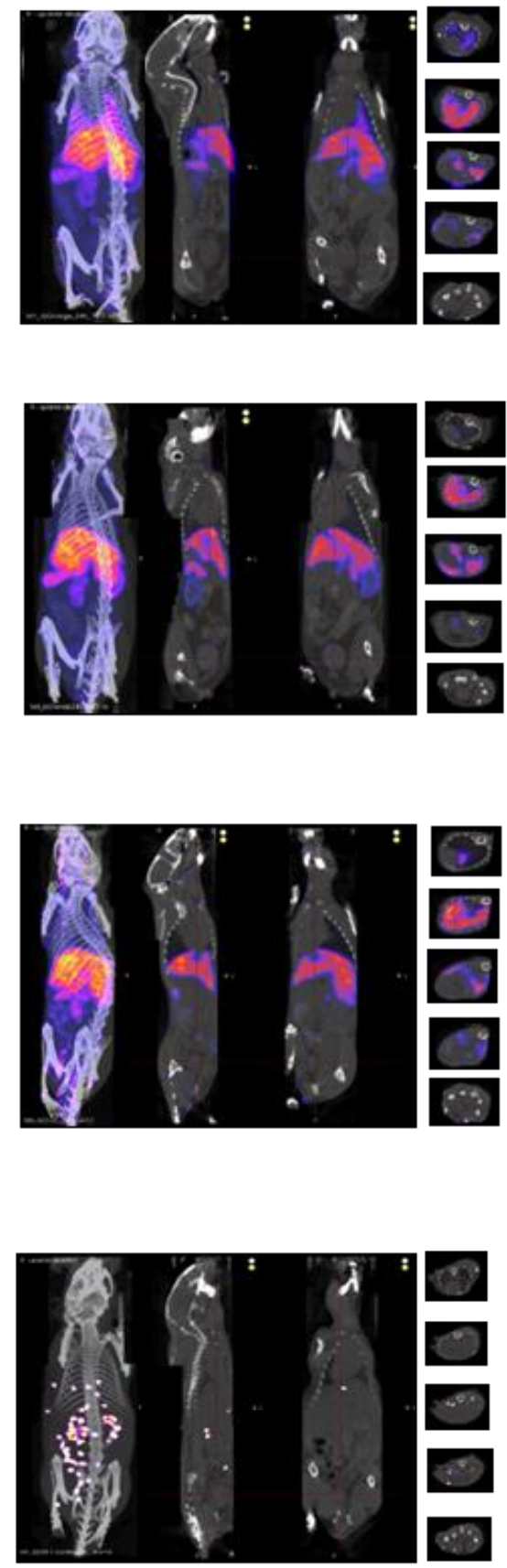

\section{$50 \%$ ID/g}

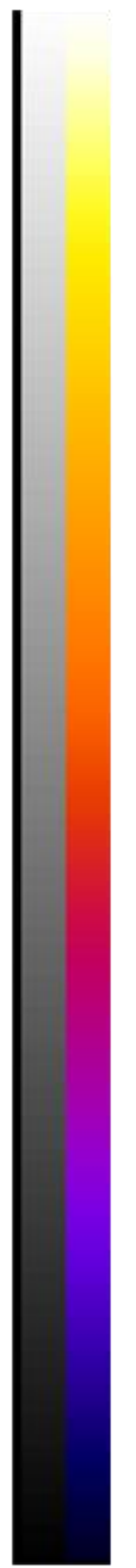

$0 \% \mathrm{ID} / \mathrm{g}$
3

Figure S9: Biodistribution of the three types of GO ([111/n]l-GO-DOTA, [ $\left.{ }^{111} \mathrm{In}\right] \mathrm{s}-\mathrm{GO}-\mathrm{DOTA}$ and [ $\left.{ }^{111} \mathrm{In}\right]$ us-GO-DOTA) compared to control [111 In]DOTA at $1 \mathrm{~h}$ and $24 \mathrm{~h}$ in a second batch of mice. From left to right (whole body maximum intensity projections (MIP), sagittal, coronal and transverse views). SPECT/CT images expressed as \% of injected dose per gram of tissues (doses were decay corrected at the second time point). Interactive 3D images (of MIPs) are available online. 

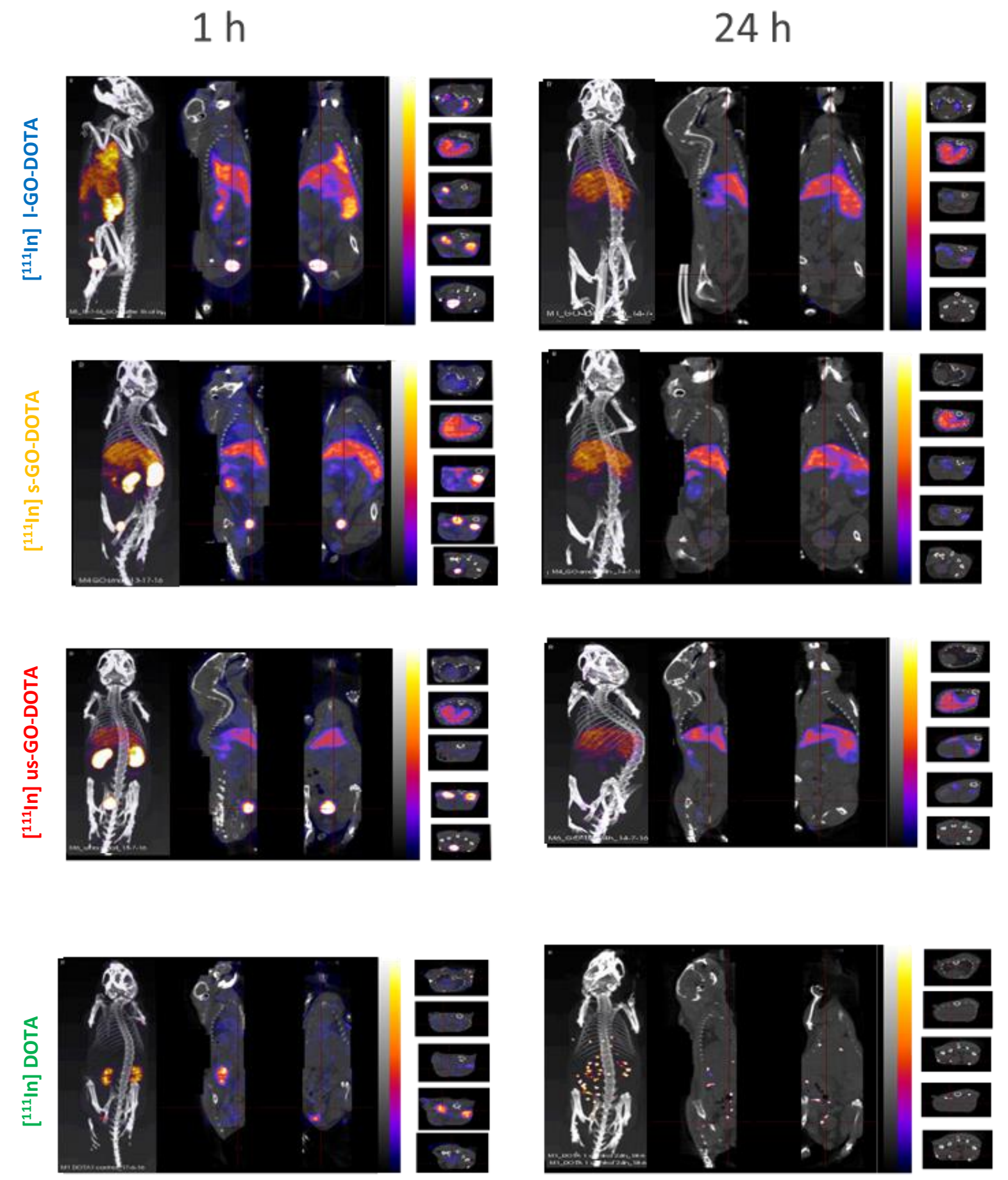

Figure S10: Biodistribution of the three types of GO ([111In]l-GO-DOTA, [111/n]s-GO-DOTA and [111/n]us-GO-DOTA) compared to control [ ${ }^{111} \mathrm{I}$ ]DOTA at $1 \mathrm{~h}$ and $24 \mathrm{~h}$ in a second batch of mice. From left to right (whole body maximum intensity projections (MIP), sagittal, coronal and transverse views). Data here are expressed in normalized $\mathrm{MBq}$ for decay and differences in injected doses between mice. Interactive 3D images (of MIPs) are available online. 
6
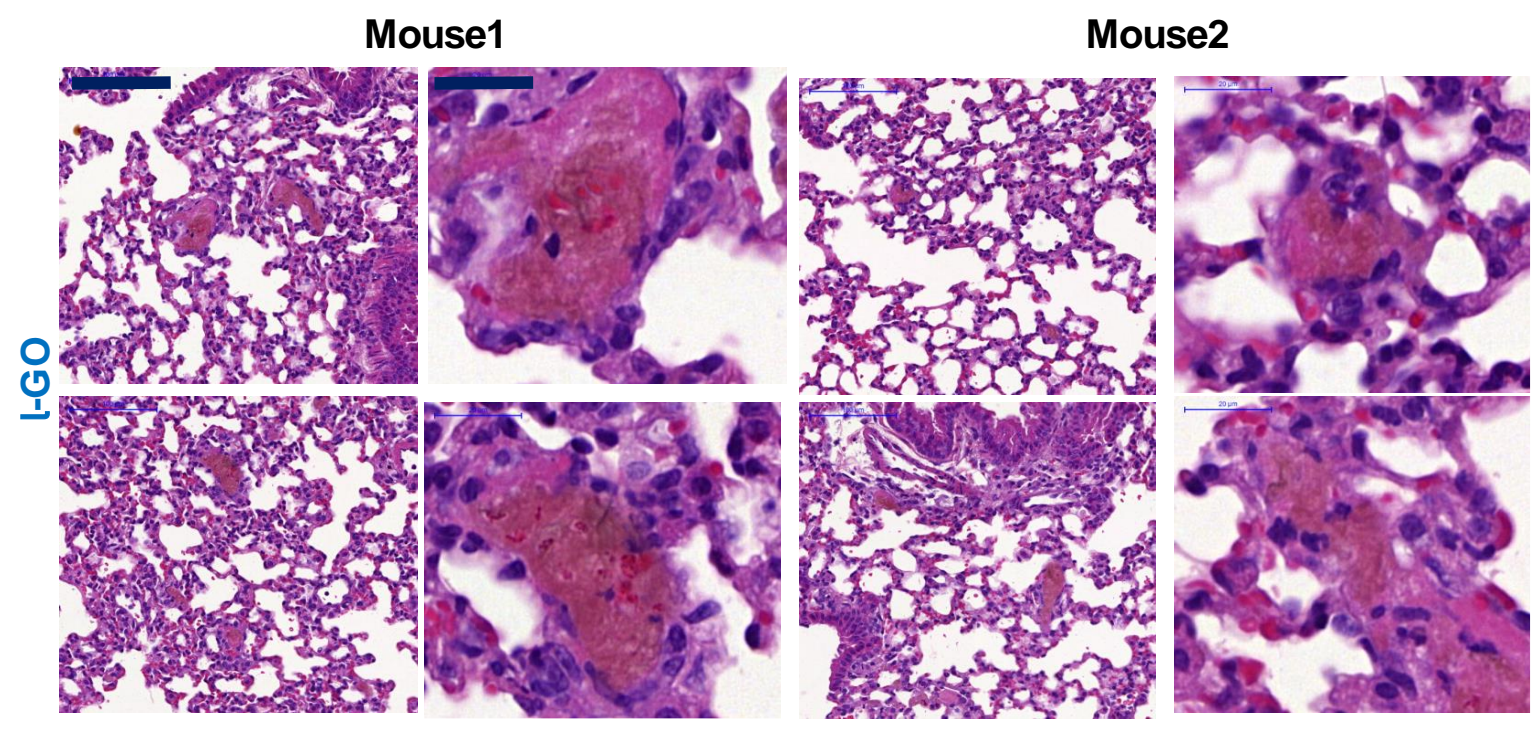

Figure S11: Effect of l-GO-DOTA on lung. Haematoxylin and eosin stained lung sections $(5 \mu \mathrm{m}$ thick) after injection of l-GO-DOTA after $24 \mathrm{~h}$ of two different mice. Scale bars for the images on the left are $100 \mu \mathrm{m}$ while those on the right are $20 \mu \mathrm{m}$. These images are captured at different locations compared to those in Figure 5. 


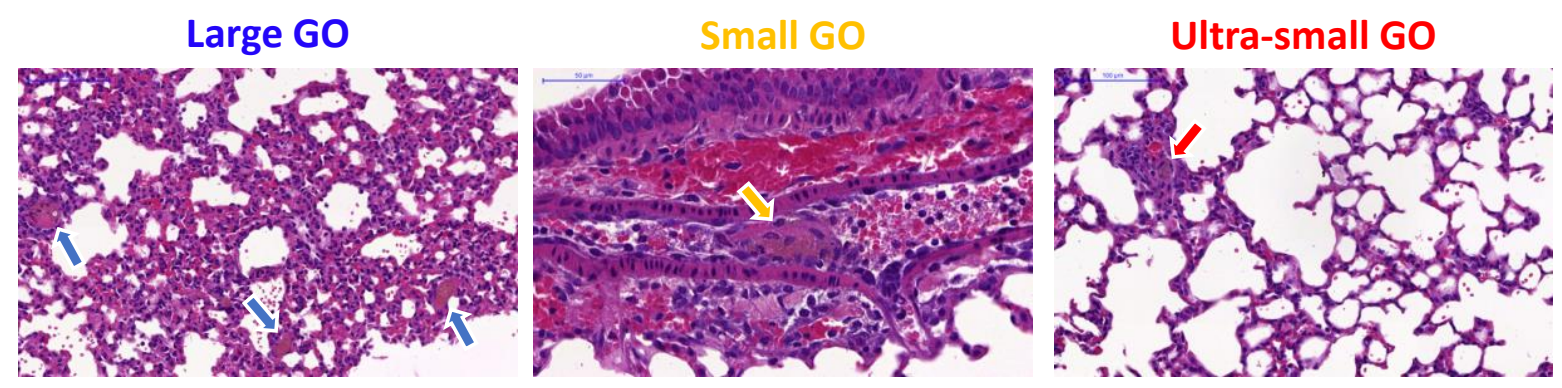

Figure S12: Effect of I-GO-DOTA, s-GO-DOTA, us-GO-DOTA on lung compared to control $5 \%$ dextrose. Haematoxylin and eosin stained lung sections (5 $\mu \mathrm{m}$ thick) after injection of l-GO-DOTA, s-GO-DOTA, us-GO-DOTA and $5 \%$ dextrose (negative control) after $24 \mathrm{~h}$ of two different mice for each material. Scale bars for the images on the left are $100 \mu \mathrm{m}$ while those on the left are $20 \mu \mathrm{m}$. 

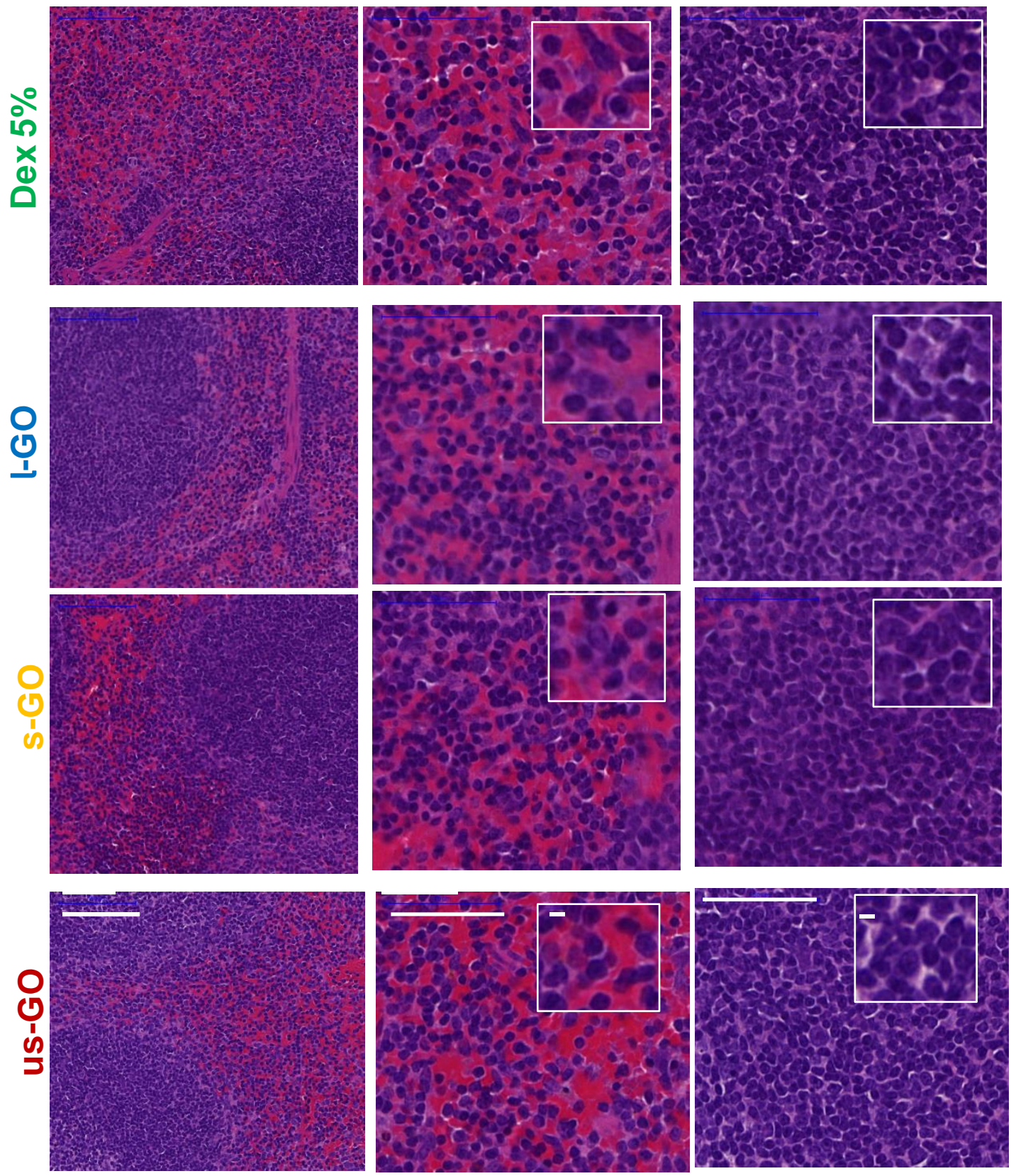

Figure S13: Effect of I-GO-DOTA, s-GO-DOTA, us-GO-DOTA on spleen structure compared to control $5 \%$ dextrose. Haematoxylin and eosin stained spleen sections (5 $\mu \mathrm{m}$ thick) after injection of I-GO-DOTA, s-GO-DOTA, us-GO-DOTA and 5\% dextrose (negative control) after 24h of first set of mice. No evidence of histopathology was determined in the red pulp (second panel) and the white pulp (third panel) in any of the samples as compared to the controls. Each point represents a different mouse. Scale bars for the images on the left are $100 \mu \mathrm{m}$ while those in the middle and last panels are $50 \mu \mathrm{m}$. The insets are $1 \mu \mathrm{m}$. 

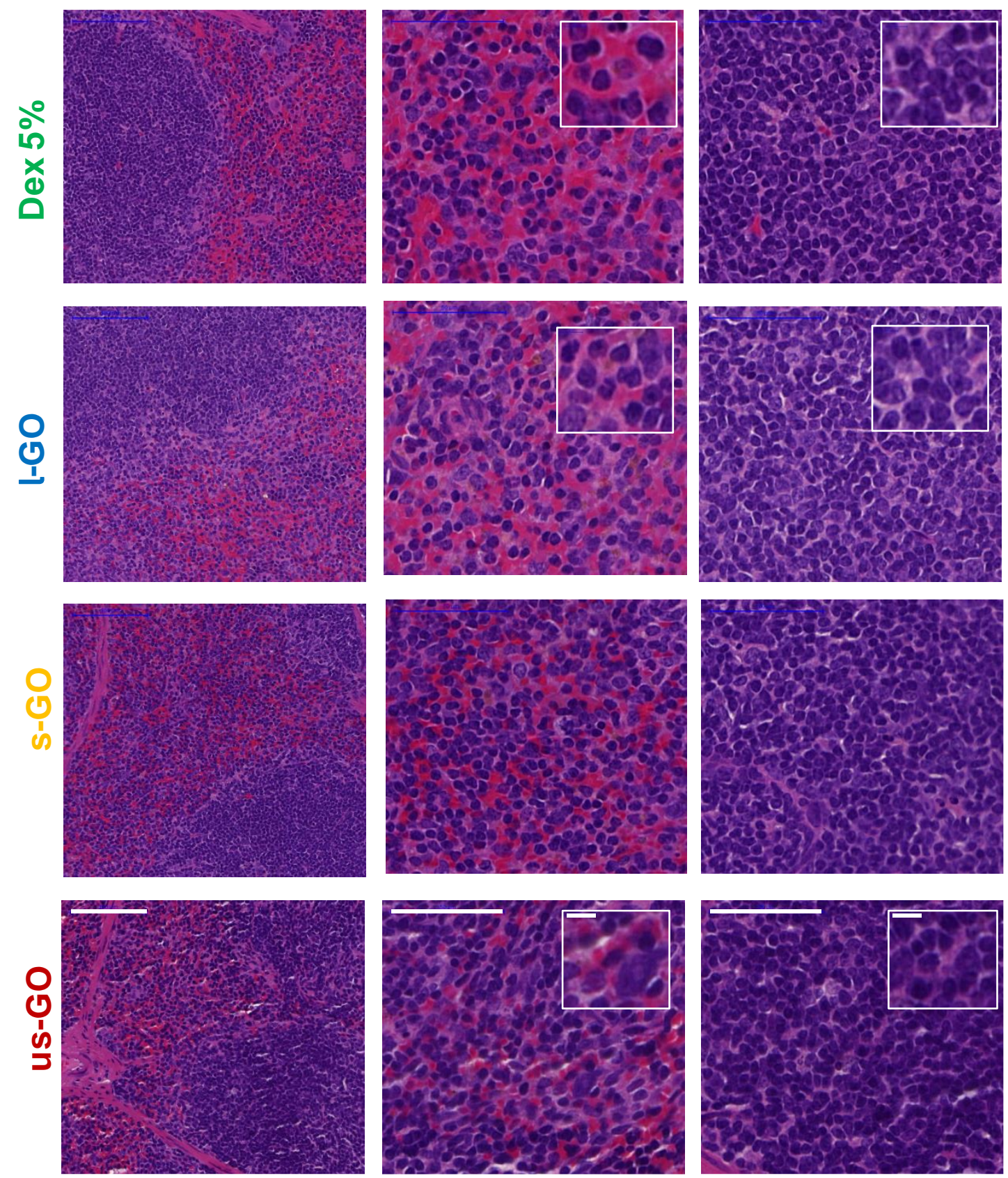

Figure S14: Effect of I-GO-DOTA, s-GO-DOTA, us-GO-DOTA on spleen structure compared to control $5 \%$ dextrose. Haematoxylin and eosin stained spleen sections (5 $\mu \mathrm{m}$ thick) after injection of I-GO-DOTA, s-GO-DOTA, us-GO-DOTA and 5\% dextrose (negative control) after 24h of second set of mice. No evidence of histopathology was determined in the red pulp (second panel) and the white pulp (third panel) in any of the samples as compared to the controls. Scale bars for the images on the left are $100 \mu \mathrm{m}$ while those in the middle and last panels are $50 \mu \mathrm{m}$,. The insets are $1 \mu \mathrm{m}$. 

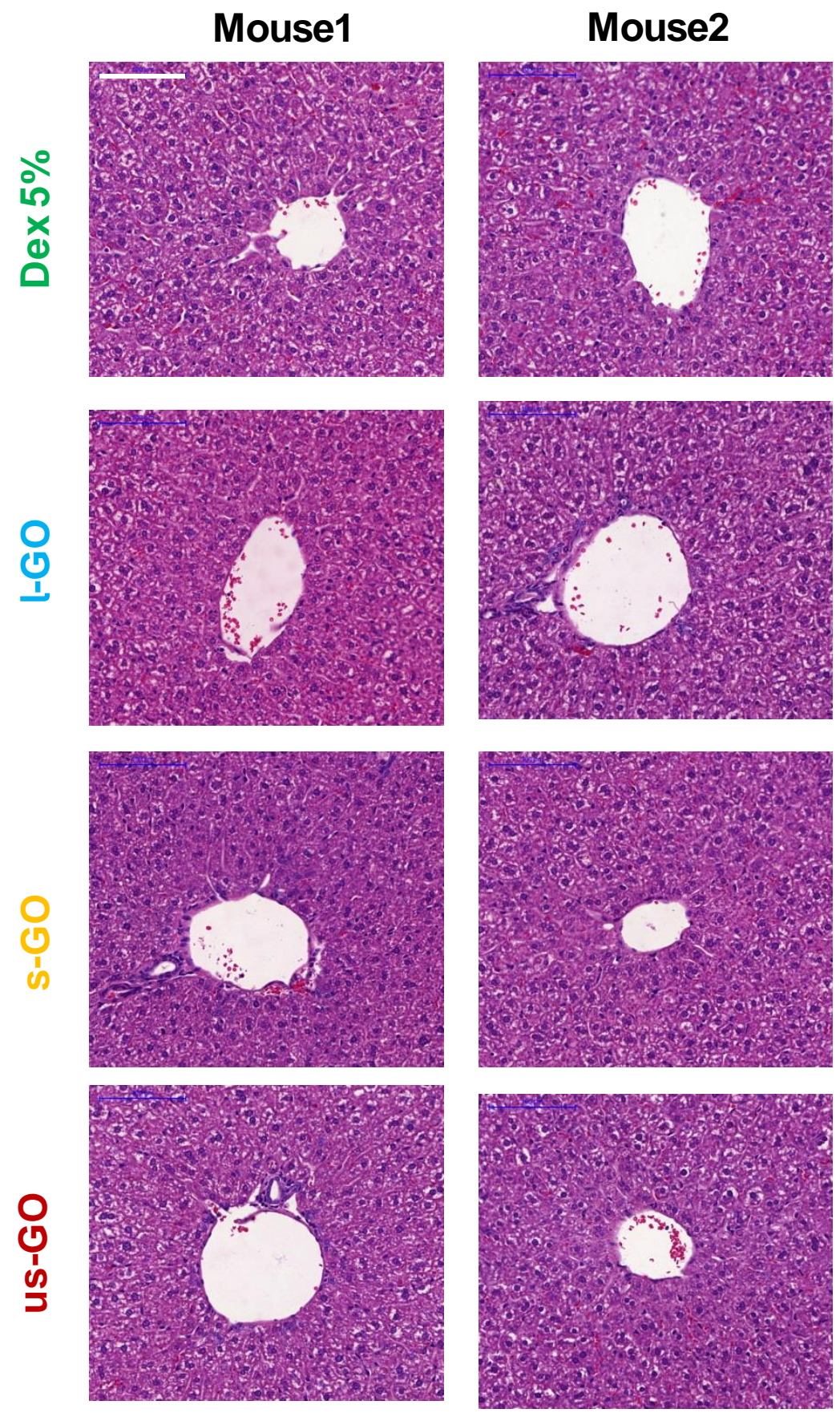

Figure S15: Effect of I-GO-DOTA, s-GO-DOTA, us-GO-DOTA on liver structure compared to control $5 \%$ dextrose. Haematoxylin and eosin stained liver sections (5 $\mu \mathrm{m}$ thick) after injection of I-GO-DOTA, s-GO-DOTA, us-GO-DOTA and $5 \%$ dextrose (negative control) after $24 \mathrm{~h}$. No evidence of histopathology was determined in the livers in any of the samples as compared to the controls. Scale bars for the images are $100 \mu \mathrm{m}$. 


\section{Mouse1}
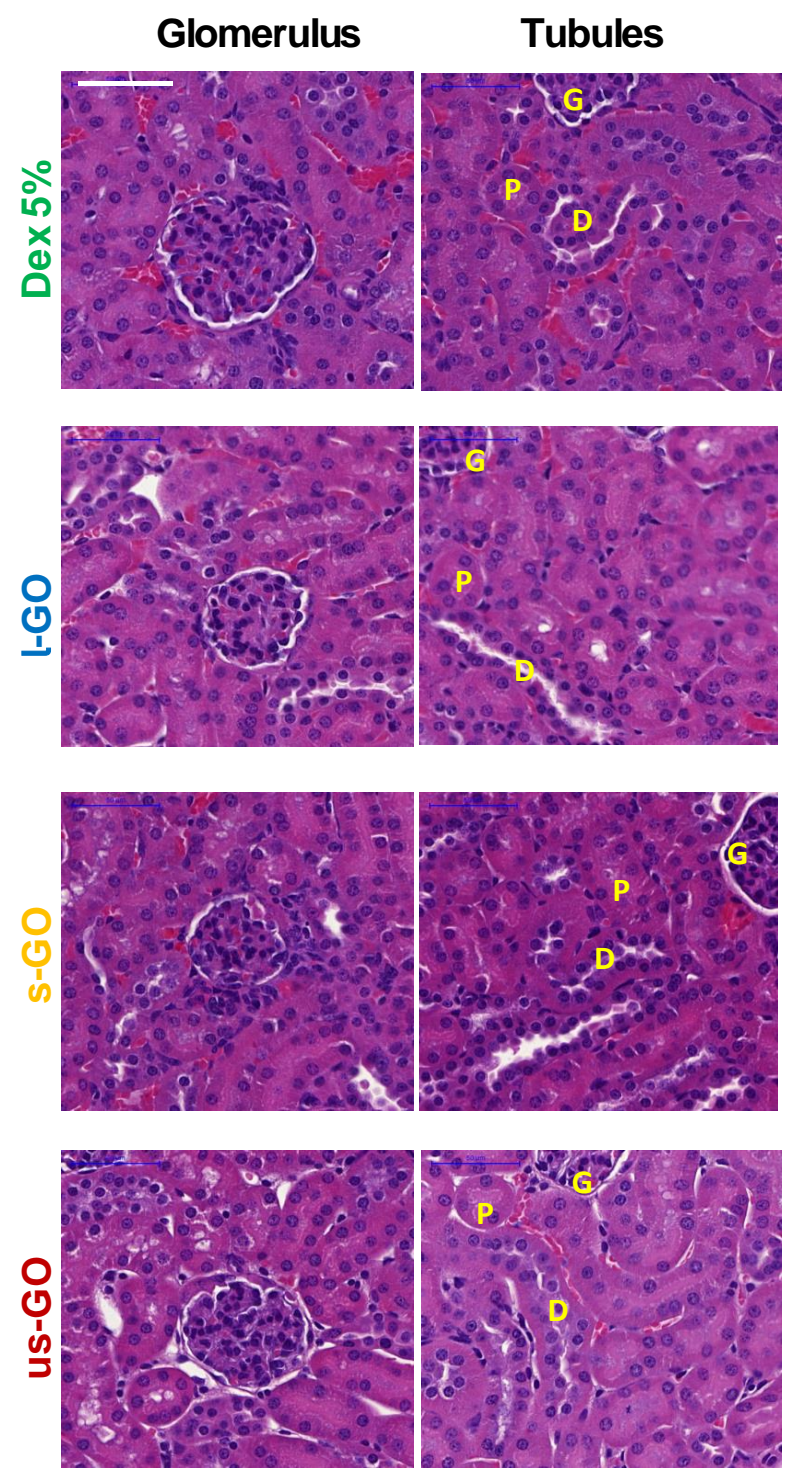

Tubules
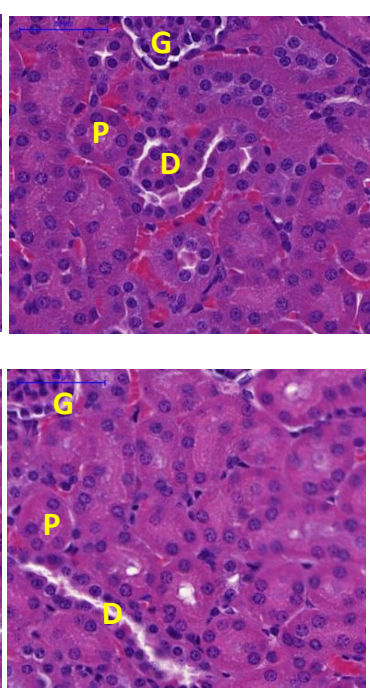

Mouse2
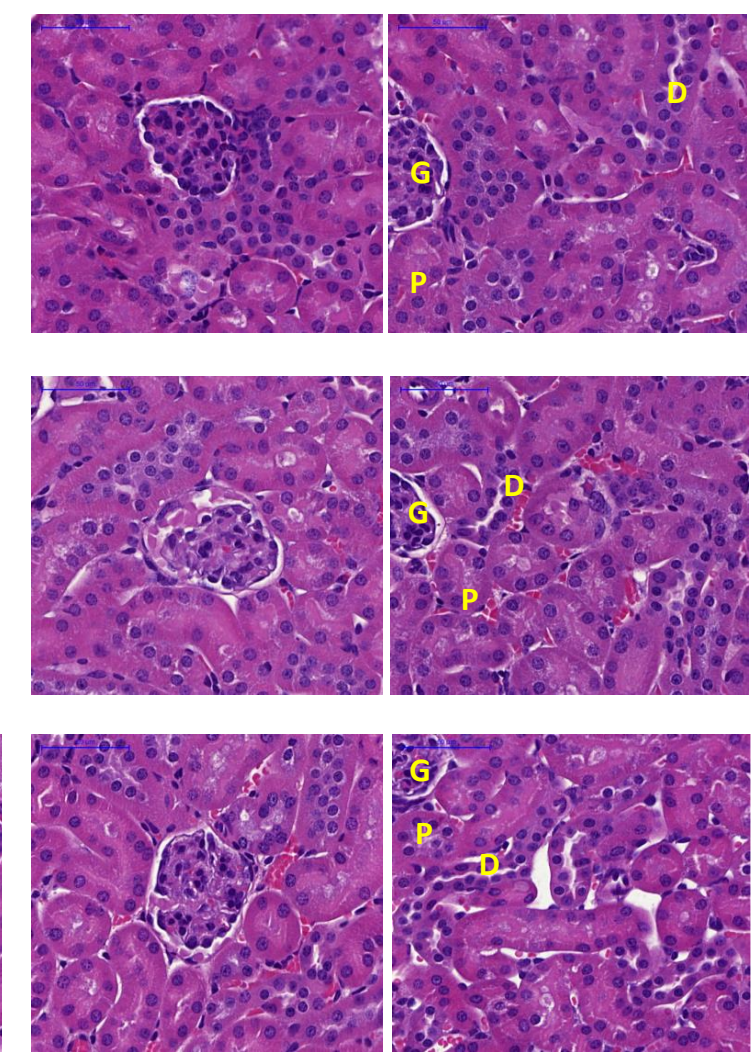

Tubules
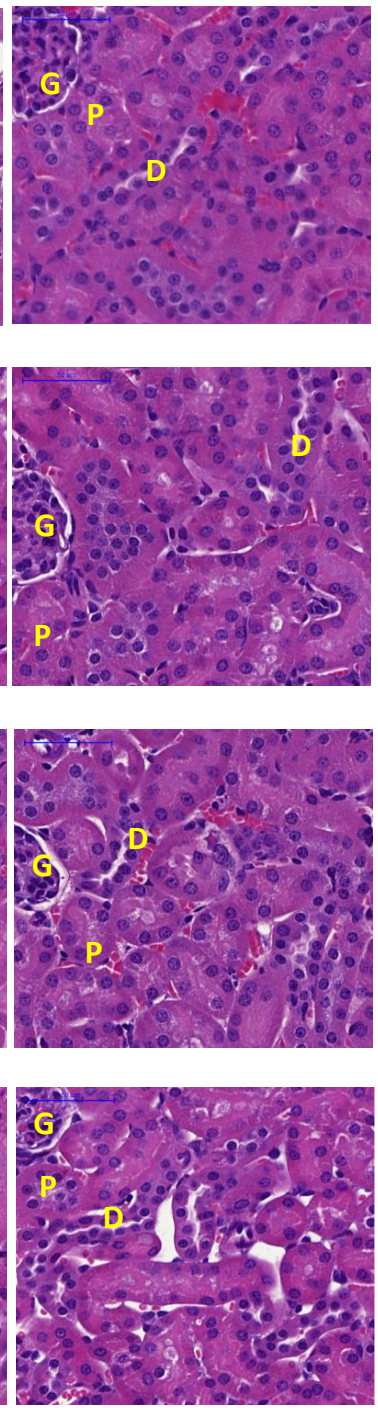

Figure S16: Effect of I-GO-DOTA, s-GO-DOTA, us-GO-DOTA on kidney structure compared to control $5 \%$ dextrose. Haematoxylin and eosin stained kidney sections (5 $\mu \mathrm{m}$ thick) after injection of l-GO-DOTA, s-GO-DOTA, us-GO-DOTA and 5\% dextrose (negative control) after 24h. No evidence of histopathology was determined in the kidney glomerular or tubular regions as compared to the controls. Scale bars for the images are $50 \mu \mathrm{m}$. G: glomerulus, P: proximal convoluted tubule and $\mathrm{D}$ : distal convoluted tubule. 


\section{Supporting Tables}

2 Table S1: Summary of the physicochemical characteristics of GO materials used herein. Measured 3 sizes are expressed as means and ranges. Full characterisation was published in previous works 4 (37, 48-50).

5

\begin{tabular}{|c|c|c|c|c|}
\hline Parameter & Method & tGO & $s-G O$ & us-GO \\
\hline Lateral & TEM & $10 \mu \mathrm{m}[1-30 \mu \mathrm{m}]$ & $450 \mathrm{~nm}[0.2-1 \mu \mathrm{m}]$ & $122 \mathrm{~nm}[10-550 \mathrm{~nm}]$ \\
\hline Dimension & $A F M$ & $21 \mu \mathrm{m}[10-30 \mu \mathrm{m}]$ & $74 \mathrm{~nm}[29-369 \mathrm{~nm}]$ & $69 \mathrm{~nm}[30-300 \mathrm{~nm}]$ \\
\hline Thickness & $A F M$ & $1.8 \mathrm{~nm}[1-6 \mathrm{~nm}]$ & $2 \mathrm{~nm}[0.5-12 \mathrm{~nm}]$ & $1 \mathrm{~nm}[0.4-2.5 \mathrm{~nm}]$ \\
\hline \multirow{2}{*}{$\begin{array}{l}\text { Colloidal } \\
\text { properties }\end{array}$} & $D L S$ & $\begin{array}{c}3229 \pm 837 \mathrm{~nm} \\
(\mathrm{PDI}=1.000 \pm 0.000)\end{array}$ & $\begin{array}{c}155.5 \pm 3.5 \mathrm{~nm} \\
(\mathrm{PDI}=0.231 \pm 0.016)\end{array}$ & $\begin{array}{c}54.6 \pm 0.7 \mathrm{~nm} \\
(\mathrm{PDI}=0.344 \pm 0.008)\end{array}$ \\
\hline & $\zeta$-potential & $-61.7 \pm 1.8 \mathrm{mV}$ & $-55.0 \pm 0.8 \mathrm{mV}$ & $-54.8 \pm 0.1 \mathrm{mV}$ \\
\hline$I(D) / I(G)$ & Raman & $1.30 \pm 0.04$ & $1.35 \pm 0.02$ & $1.34 \pm 0.03$ \\
\hline $\begin{array}{l}\text { Elemental } \\
\text { composition }\end{array}$ & $X P S$ & C: $68.6 \%, 0: 31.4 \%$ & C: $68.5 \%, 0: 31.5 \%$ & C: $69.8 \%, 0: 30.2 \%$ \\
\hline Parameter & Method & -GO-DOTA & S-GO-DOTA & Us-GO-DOTA \\
\hline Lateral & TEM & $2 \mu \mathrm{m}[0.2-5 \mu \mathrm{m}]$ & $354 \mathrm{~nm}[29-1434 \mathrm{~nm}]$ & $160 \mathrm{~nm}[10-538 \mathrm{~nm}]$ \\
\hline Dimension & $A F M$ & $0.36 \mu \mathrm{m}[0.1-3.5 \mu \mathrm{m}]$ & $91 \mathrm{~nm}[30-800 \mathrm{~nm}]$ & $49 \mathrm{~nm}[30-480 \mathrm{~nm}]$ \\
\hline Thickness & AFM & $1.9 \mathrm{~nm}[0.3-51.2 \mathrm{~nm}]$ & $2.5 \mathrm{~nm}[0.2-34.3 \mathrm{~nm}]$ & $2.6 \mathrm{~nm}[0.2-48.0 \mathrm{~nm}]$ \\
\hline$I(D) / I(G)$ & Raman & $1.34 \pm 0.05$ & $1.33 \pm 0.03$ & $1.34 \pm 0.02$ \\
\hline $\begin{array}{l}\text { Colloidal } \\
\text { stability }\end{array}$ & $\zeta$-potential & $-31.1 \pm 0.8 \mathrm{mV}$ & $-36.7 \pm 0.4 \mathrm{mV}$ & $-38.8 \pm 0.9 \mathrm{mV}$ \\
\hline $\begin{array}{l}\text { Elemental } \\
\text { composition }\end{array}$ & $X P S$ & $\begin{array}{c}\text { C: } 71.4 \%, 0: 27.5 \%, \\
N: 1.1 \%\end{array}$ & $\begin{array}{c}\text { C: } 69.6 \%, 0: 29.0 \%, \\
N: 1.4 \%\end{array}$ & $\begin{array}{c}\text { C: } 68.9 \%, \text { O: } 29.7 \%, \\
\text { N: } 1.4 \%\end{array}$ \\
\hline
\end{tabular}


1 Table S2: First-order pharmacokinetic parameters of all three [ $\left.{ }^{111} \mathrm{In}\right]$ GO-DOTA materials compared 2 to control [111In] DOTA post intravenous exposure.

3

$\begin{array}{ccccc}t_{1 / 2 \alpha} & t_{1 / 2 \beta} & A U C & V_{s s} & C L \\ \min & h & m g \cdot h / L & m l & \mathrm{ml} / \mathrm{h}\end{array}$

[111 In] I-GO-DOTA $\quad 21.2 \pm 15.5 \quad 7.2 \pm 1.8 \quad 28.4 \pm 11.8 \quad 14.5 \pm 2.9 \quad 3.2 \pm 1.3$

$22.9 \pm 4.9 \quad 5.4 \pm 3.2 \quad 22.9 \pm 4.9 \quad 5.9 \pm 1.6 \quad 3.1 \pm 1.2$

[111 $\mathrm{In}$ ] us-GO-DOTA $\quad 21.9 \pm 5.8 \quad 9.8 \pm 3.6 \quad 21.9 \pm 5.8 \quad 13.2 \pm 5.0 \quad 2.2 \pm 0.8$

4

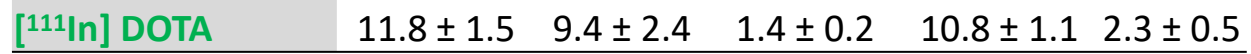


2 Table S3: Remaining amounts of all three [111In] GO-DOTA materials (compared to [111In] DOTA

3 control) in the blood ( $\mu \mathrm{g}$ per $\mathrm{ml}$ of blood), quantified by $\mathrm{\gamma}$-counting ( $\mathrm{n}=4$ per condition).

\begin{tabular}{|c|c|c|}
\hline Sample & $\begin{array}{c}1 \mathrm{~h} \\
\mu \mathrm{g} / \mathrm{ml}\end{array}$ & $\begin{array}{c}24 \mathrm{~h} \\
\mu \mathrm{g} / \mathrm{ml}\end{array}$ \\
\hline$\left[{ }^{111} \mid n\right]$ I-GO-DOTA & $1.6 \pm 3.7$ & $0.41 \pm 0.3$ \\
\hline$\left[{ }^{111} \mid n\right]$ s-GO-DOTA & $3.6 \pm 2.6$ & $0.41 \pm 0.2$ \\
\hline [111 In] us-GO-DOTA & $3.5 \pm 4.1$ & $0.42 \pm 0.2$ \\
\hline [111/n] DOTA & $0.1 \pm 0.1$ & $0.01 \pm 0.0$ \\
\hline
\end{tabular}

5

6 
2 Table S4: SPECT/CT quantification showing the remaining amounts of all three materials 3 compared to control in the body after $24 \mathrm{~h}, \mathrm{n}=2$ per condition.

4

\begin{tabular}{|c|c|}
\hline Sample & $\%$ ID after $24 \mathrm{~h}$ \\
\hline [111/n] I-GO-DOTA & $50.8 \pm 17.4$ \\
\hline [111/n] s-GO-DOTA & $36.5 \pm 2.7$ \\
\hline$\left[{ }^{111} \mid \mathrm{n}\right]$ us-GO-DOTA & $38.1 \pm 5.3$ \\
\hline [111 ln] DOTA & $5.1 \pm 1.9$ \\
\hline
\end{tabular}

